\title{
IL-1ß suppression of VE-cadherin transcription underlies sepsis-induced inflammatory lung injury
}

\author{
Shiqin Xiong, ${ }^{1,2}$ Zhigang Hong, ${ }^{1,2}$ Long Shuang Huang, ${ }^{1,2}$ Yoshikazu Tsukasaki, ${ }^{1,2}$ Saroj Nepal, ${ }^{1,2}$ Anke Di, ${ }^{1,2}$ Ming Zhong, ${ }^{1,2}$ \\ Wei Wu, ${ }^{1,2}$ Zhiming Ye, ${ }^{3}$ Xiaopei Gao, ${ }^{1,2}$ Gadiparthi N. Rao, ${ }^{4}$ Dolly Mehta, ${ }^{1,2}$ Jalees Rehman, ${ }^{1,2}$ and Asrar B. Malik ${ }^{1,2}$ \\ 'Department of Pharmacology and Regenerative Medicine and ${ }^{2}$ Center for Lung and Vascular Biology, University of Illinois College of Medicine, Chicago, Illinois, USA. ${ }^{3}$ Department of Nephrology, Guangdong \\ Provincial People's Hospital, Guangdong Academy of Medical Sciences, Guangzhou, China. ${ }^{4}$ Department of Physiology, University of Tennessee Health Science Center, Memphis, Tennessee, USA.
}

\begin{abstract}
Unchecked inflammation is a hallmark of inflammatory tissue injury in diseases such as acute respiratory distress syndrome (ARDS). Yet the mechanisms of inflammatory lung injury remain largely unknown. Here we showed that bacterial endotoxin lipopolysaccharide (LPS) and cecal ligation and puncture-induced (CLP-induced) polymicrobial sepsis decreased the expression of transcription factor CAMP response element binding (CREB) in lung endothelial cells. We demonstrated that endothelial CREB was crucial for VE-cadherin transcription and the formation of the normal restrictive endothelial adherens junctions. The inflammatory cytokine IL-1 $\beta$ reduced cAMP generation and CREB-mediated transcription of VEcadherin. Furthermore, endothelial cell-specific deletion of CREB induced lung vascular injury whereas ectopic expression of CREB in the endothelium prevented the injury. We also observed that rolipram, which inhibits type 4 cyclic nucleotide phosphodiesterase-mediated (PDE4-mediated) hydrolysis of CAMP, prevented endotoxemia-induced lung vascular injury since it preserved CREB-mediated VE-cadherin expression. These data demonstrate the fundamental role of the endothelial CAMP-CREB axis in promoting lung vascular integrity and suppressing inflammatory injury. Therefore, strategies aimed at enhancing endothelial CREB-mediated VE-cadherin transcription are potentially useful in preventing sepsis-induced lung vascular injury in ARDS.
\end{abstract}

\section{Introduction}

Sepsis-induced acute respiratory distress syndrome (ARDS), a syndrome characterized by acute respiratory failure and intractable hypoxemia, is the result of pulmonary vascular injury and edema formation $(1,2)$. The absence of any targeted drug treatment is in part due to the complex nature of sepsis-induced lung inflammatory response and involvement of multiple cell types (endothelial and inflammatory phagocytic cells) in disease pathogenesis and progression $(1,3-5)$. It has recently become apparent that activation of inflammasomes such as NLRP3 in macrophages (6) and endothelial cells (7) and generation of the proinflammatory cytokines IL-1 $\beta$ and IL-18 play a fundamental role in the mechanism of inflammatory lung injury (6-9). IL-1 $\beta$ functions in a paracrine and autocrine manner in the inflammatory milieu through activation the plasmalemmal receptor IL-1R1 expressed in multiple cell types, including the endothelium $(10,11)$. Studies showed that IL-1 $\beta$ disrupted vascular integrity (11) pointing to its involvement in vascular injury; however, the mechanisms of its action and role in inflammatory lung injury have not been addressed.

Severely increased lung vascular permeability is a primary underlying cause of sepsis-induced inflammatory lung injury (12). Endothelial permeability in vessels is regulated by homotypic

Conflict of interest: The authors have declared that no conflict of interest exists. Copyright: () 2020, American Society for Clinical Investigation.

Submitted: January 31, 2020; Accepted: April 9, 2020; Published: June 8, 2020.

Reference information: J Clin Invest. 2020;130(7):3684-3698.

https://doi.org/10.1172/JCl136908. interaction of VE-cadherin, a central component of endothelial adherens junctions (AJs) that mediate endothelial junctional integrity and lung fluid balance $(12,13)$. Endocytosis and degradation of VE-cadherin through phosphorylation- and ubiquitinationdependent mechanisms $(14,15)$ in response to inflammatory stimuli lead to increased endothelial permeability through impaired VE-cadherin interactions $(11,15,16)$. The resulting disruption of AJs is a primary cause of inflammatory lung injury (17), but little is known about the underlying transcriptional mechanisms in the endothelium that promote breakdown of AJs and induce inflammatory injury. Here, we show that IL-1 $\beta$ signaling in endothelial cells suppressed $V E$-cadherin transcription by inactivation of the transcription factor cAMP response element binding (CREB). Furthermore, genetically restoring endothelial CREB expression and its transcriptional activity in lung endothelial cells of mice was sufficient to reverse sepsis-induced inflammatory lung injury, pointing to a potential therapeutic target.

\section{Results}

$C R E B$ regulates VE-cadherin transcription. To address the basis of lung vascular injury induced by LPS in mice, we first calibrated the endotoxemia model by performing a time course of lung vascular permeability changes, and observed endothelial permeability peaking after LPS for 1 day, and then gradually decreasing during the repair period over 5 days (Figure 1A). Protein expression of CREB and VE-cadherin in lungs and freshly isolated endothelial cells from these mice were coincidently decreased and also returned to basal levels 5 days after LPS challenge (Figure 1, B and 
C). Corresponding changes in mRNA levels of CREB and VE-cadherin were seen during the injury and repair periods (Figure 1D), suggesting a key role for a CREB transcriptional mechanism in mediating the changes in endothelial permeability. Furthermore, similar changes in CREB and VE-cadherin expression were observed in the altogether different polymicrobial sepsis model cecal ligation and puncture (CLP) (Supplemental Figure 1, A-C; supplemental material available online with this article; https:// doi.org/10.1172/JCI136908DS1).

We next determined whether CREB regulated VE-cadherin transcription and thereby promoted lung endothelial barrier function. CREB is known to bind preferentially the palindromic (TGACGTCA) or half-site (TGAC/G) cyclic AMP response elements (CREs) (18). The 2.5-kb mouse and human VE-cadherin promoter regions contain several of these conserved CREs for CREB binding (Supplemental Figure 2). Depletion of CREB by siRNA in mouse lung microvascular endothelial cells (mLMVECs) decreased VE-cadherin expression (Figure 1E) while upregulating the expression of CREB increased VE-cadherin (Figure 1F). ChIP assay showed that CREB bound both CRE2 and CRE5 within $V E$-cadherin promoter (Figure $1 G$ ). Luciferase reporter assays also demonstrated that CRE2 and CRE5 binding sites were essential for CREB-induced $V E$-cadherin transcription (Figure $1 \mathrm{H}$ ).

$I L-1 \beta$ impairs CREB-mediated VE-cadherin transcription to induce endothelial injury. As IL-1 $\beta$ generation by immune cells via activation of inflammatory caspases and the inflammasome NLRP3 is a key mechanism of inflammatory tissue damage (6, $8)$, we evaluated the possible role of IL- $1 \beta$ signaling in endothelial cells as a critical mediator of lung vascular injury. Deletion of IL-1 $\beta$ receptor IL-1R1 in endothelial cells prevented the reduction in CREB and VE-cadherin expression in lung endothelial cells after LPS challenge (Supplemental Figure 3A). Lung vascular injury was also suppressed in $I L-1 R 1^{-/-}$mice (Supplemental Figure $3 \mathrm{~B}$ ) and there was no lung edema as compared with WT mice (Supplemental Figure 3C).

The notion that IL-1 $/$ IL-1R1 signaling in endothelial cells is an important determinant of endotoxemia-induced lung vascular injury was also addressed in studies showing that IL-1 $\beta$ challenge itself increased endothelial permeability (Figure 2A). IL-1 $\beta$ decreased the expression of both CREB and VE-cadherin in a concentration- and time-dependent manner in mLMVECs (Figure 2, B-D) but had no effect on $\beta$-catenin expression, another key component of endothelial adherens junctions. IL-1 $\beta$ also reduced CREB and VE-cadherin expression in hLMVECs, but had no impact on expression and Ser 114 phosphorylation of endothelial nitric oxide synthase (eNOS) (Supplemental Figure 4A). Furthermore, IL-1 $\beta$ did not change CREB ubiquitination (Figure $2 \mathrm{E}$ ), ruling out CREB proteolysis and degradation as a basis of the IL-1 $\beta$ mediated lung endothelial injury.

We next addressed the signaling mechanisms of IL-1 $\beta$-mediated downregulation of CREB and VE-cadherin expression. Here we posited that activation of soluble adenylyl cyclase (sAC, or ADCY10) (19) and generation of the second messenger cAMP in endothelial cells regulated CREB expression through an autoregulatory CREB transcriptional feedback loop in which cAMP-activated CREB induces its own transcription by binding to CRE sites on CREB promoter. We observed that IL-1 $\beta$ reduced sAC activity (Figure $2 \mathrm{~F}$ ) and intracellular cAMP concentrations in mLMVECs (Figure $2 \mathrm{G}$ ) and in hLMVECs (Supplemental Figure 4B). Impaired CREB expression might be due to defective binding of CREB to conserved CREs in the promoter (Supplemental Figure 5). ChIP assays showed that CREB directly bound CRE2 and CRE6 sites within the 2.5-kb mouse CREB promoter region (Figure $2 \mathrm{H}$ ) and that IL-1 $\beta$ reduced CREB binding to the CRE2 and CRE6 sites (Figure 2I).

In other studies, we evaluated the effects of sAC knockdown on the generation of CAMP, expression changes of CREB and VE-cadherin, and permeability changes in endothelial cells. Depletion of SAC in endothelial cells reduced cAMP generation (Figure 3A) and expression of CREB and VE-cadherin (Figure 3B), and induced endothelial injury (Figure 3C).

To determine whether IL-1 $\beta$ was responsible for the reduction in VE-cadherin expression through disrupting CREB-mediated transcription, ChIP assays were carried out using anti-CREB antibody. IL-1 $\beta$ signaling disrupted the binding of CREB to CRE2 and CRE5 within the 2.5-kb VE-cadherin promoter region (Figure 3D). A reporter assay also showed that IL-1 $\beta$ prevented CREB-mediated VE-cadherin transcription (Figure 3E). Furthermore, upregulation of expression of CREB in lung endothelial cells reduced IL-1 $\beta$-induced increase in endothelial permeability (Figure 3, F and $G$ ) whereas expression of CREB prevented the reduction in VE-cadherin expression induced by IL-1 $\beta$ (Figure $3 \mathrm{H}$ ). Together, these findings show the requisite role of IL- $1 \beta$ in promoting lung endothelial injury by downregulating CREB expression and CREB-mediated VE-cadherin transcription.

Increased CAMP signaling and CREB expression in endothelial cells prevents inflammatory lung injury. We next addressed whether enabling cAMP signaling would reduce inflammatory lung injury. Here we used rolipram, which selectively inhibits type 4 cyclic nucleotide phosphodiesterase-mediated (PDE4-mediated) hydrolysis of cAMP $(20,21)$. Rolipram prevented LPS-induced reduction in cAMP concentrations (Figure $4 \mathrm{~A}$ ) as well as IL-1 $\beta$ induced reduction in $\mathrm{CREB}-\mathrm{VE}-\mathrm{cadh}$ rin signaling in a concentration-dependent manner (Figure 4, B and C). Rolipram treatment of mice resulted in significantly reduced LPS-induced lung neutrophil infiltration (Figure 4, D-F) and lung vascular injury (Figure 4, G and H). Employing lung intravital imaging methods (22), we also determined the effects of rolipram on the dynamics of polymorphonuclear leukocytes (PMNs) in lung microvessels after LPS challenge. Rolipram significantly decreased PMN number and transit times in lung vessels and increased PMN velocity (Supplemental Figure 6 and Supplemental Video 1).

To address the role of endothelial CREB in regulating VEcadherin expression and the mechanism of lung vascular injury, we generated endothelial-specific expression constructs by cloning the active fragments of the mouse $V E$-cadherin promoter into the upstream of the CREB coding sequence (Supplemental Figure 7A). Employing nanoparticle liposome-mediated gene delivery $(23,24)$, we observed that the 2.5 -kb VE-cadherin promoter induced CREB expression in the mouse lung endothelium (Supplemental Figure 7, B-E). Endothelial-specific CREB expression in these mice increased VE-cadherin expression when compared with controls (Supplemental Figure 7, C-E), indicating that endothelial CREB was required for VE-cadherin transcription in the lung endothelium. 

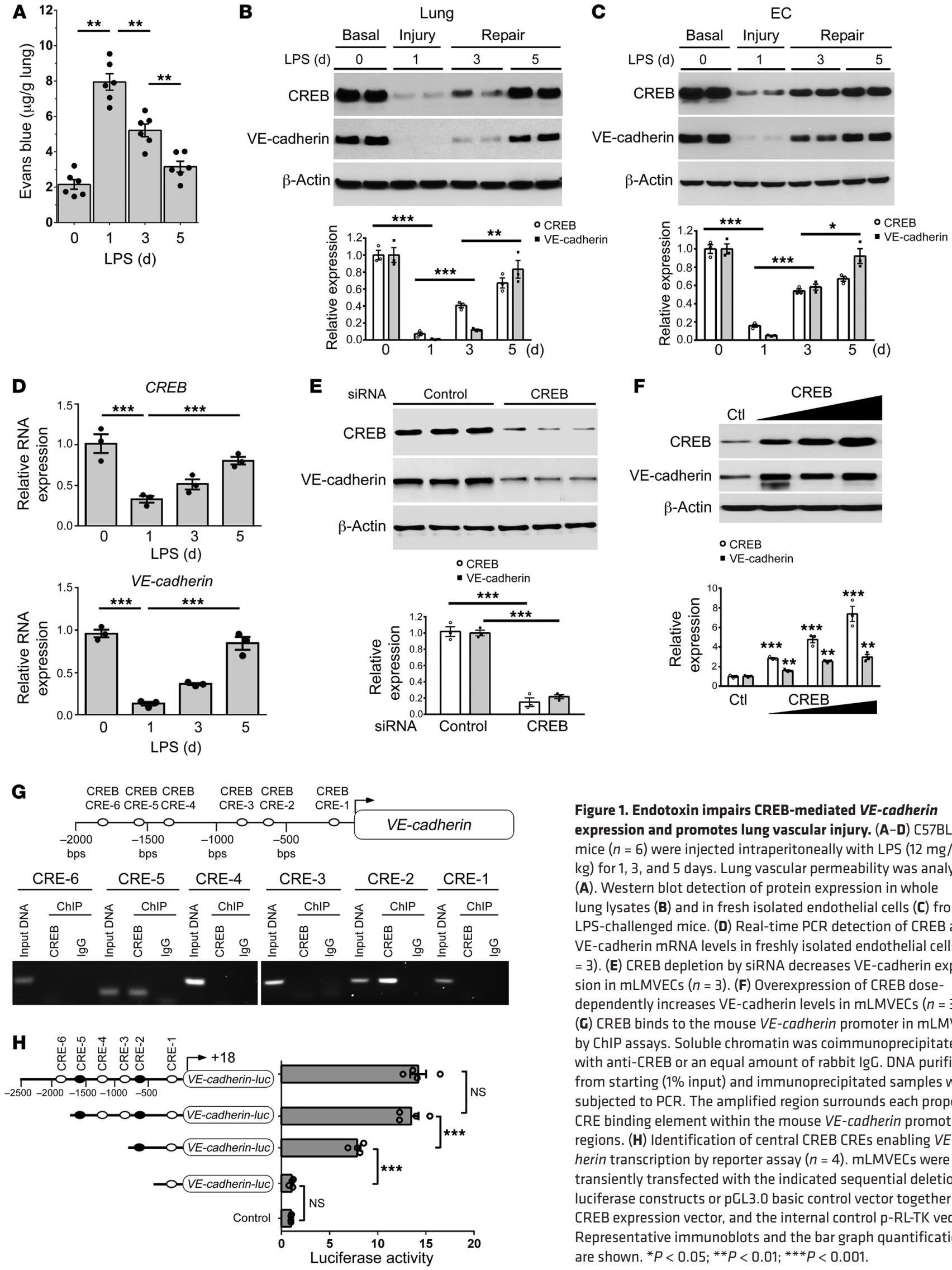

Figure 1. Endotoxin impairs CREB-mediated VE-cadherin expression and promotes lung vascular injury. (A-D) $[57 \mathrm{BL} / 6]$ mice $(n=6)$ were injected intraperitoneally with LPS ( $12 \mathrm{mg} /$ $\mathrm{kg}$ ) for 1,3 , and 5 days. Lung vascular permeability was analyzed (A). Western blot detection of protein expression in whole lung lysates (B) and in fresh isolated endothelial cells (C) from LPS-challenged mice. (D) Real-time PCR detection of CREB and VE-cadherin mRNA levels in freshly isolated endothelial cells ( $n$ = 3). (E) CREB depletion by siRNA decreases VE-cadherin expression in mLMVECs $(n=3)$. (F) Overexpression of CREB dosedependently increases VE-cadherin levels in mLMVECs $(n=3)$. (C) CREB binds to the mouse VE-cadherin promoter in mLMVECs by ChIP assays. Soluble chromatin was coimmunoprecipitated with anti-CREB or an equal amount of rabbit IgG. DNA purified from starting ( $1 \%$ input) and immunoprecipitated samples was subjected to PCR. The amplified region surrounds each proposed CRE binding element within the mouse VE-cadherin promoter regions. (H) Identification of central CREB CREs enabling VE-cadherin transcription by reporter assay $(n=4)$. MLMVECs were transiently transfected with the indicated sequential deletion luciferase constructs or pGL3.0 basic control vector together with CREB expression vector, and the internal control $p-R L-T K$ vector. Representative immunoblots and the bar graph quantification are shown. ${ }^{*} P<0.05 ;{ }^{* *} P<0.01 ;{ }^{* *} P<0.001$. 

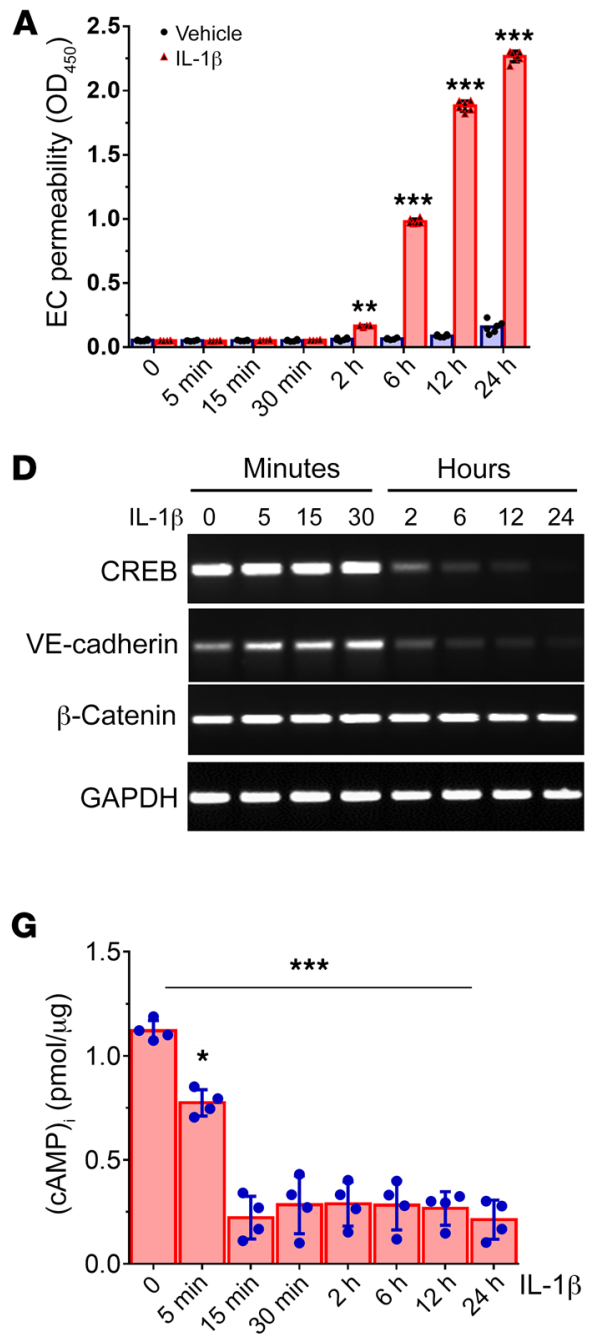

B

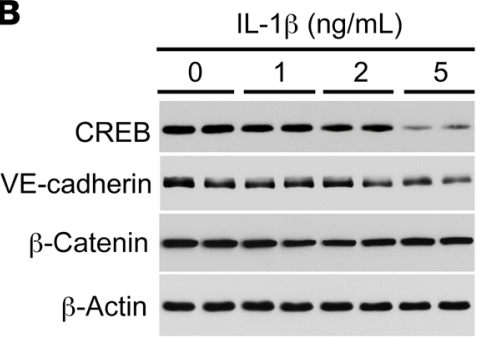

$\mathbf{E}$

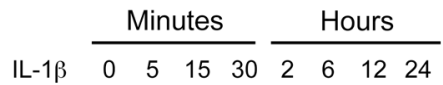

IP: CREB

IB: Ubiquitin

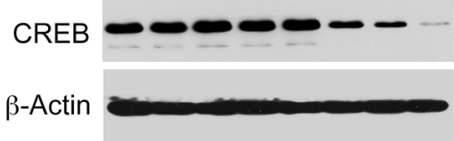

C

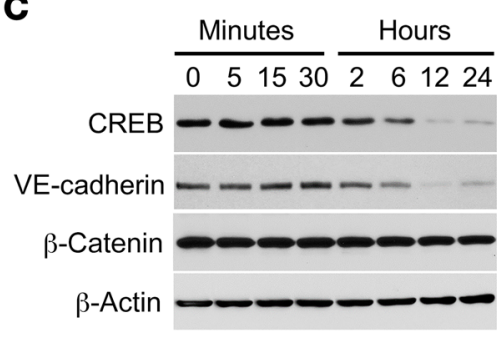

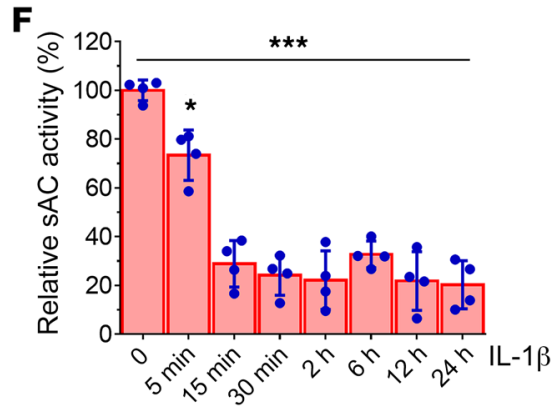

H

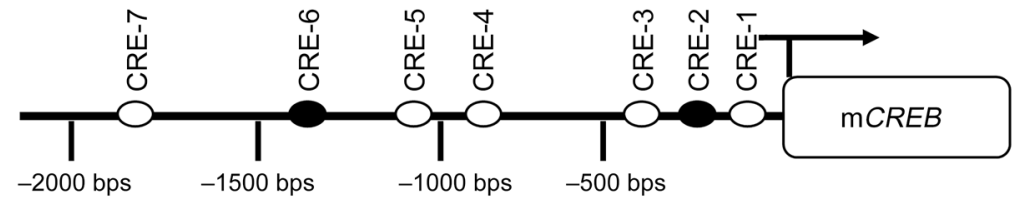

CRE-1 CRE-2 CRE-3 CRE-4 CRE-5 CRE-6 CRE-7

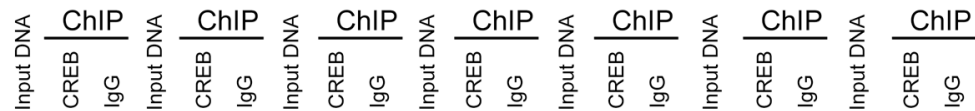

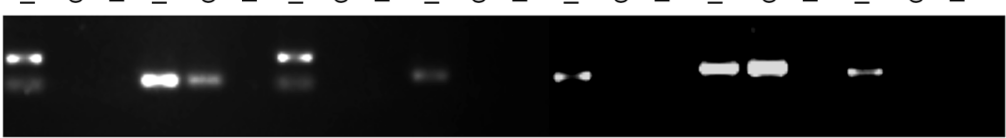

I

ChIP DNA
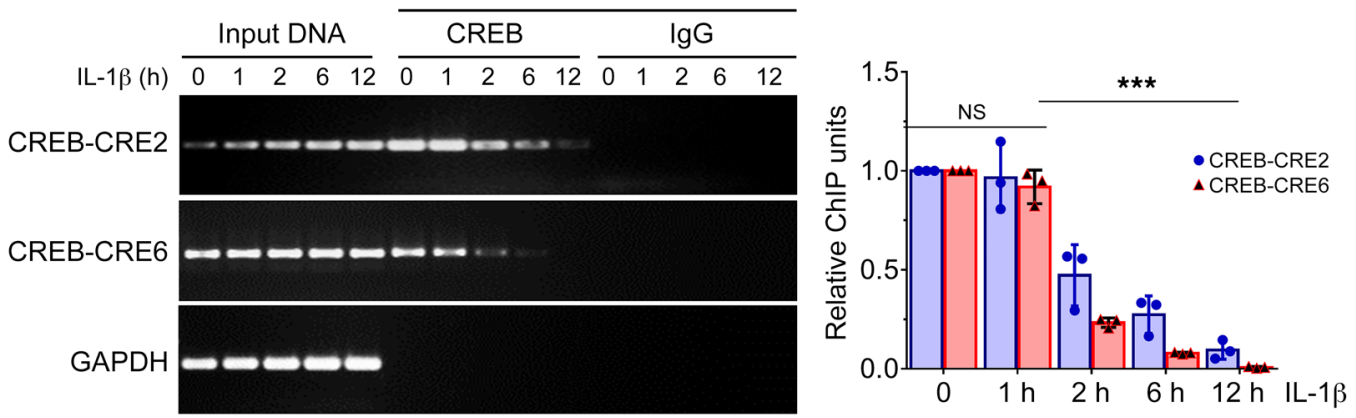

Figure 2. IL-1 $\beta$ represses CAMP-CREB signaling. (A) mLMVECs were treated with IL-1 $1(5 \mathrm{ng} / \mathrm{mL})$ or vehicle for the indicated time. Transwell permeability to HRP was measured $(n=6)$. (B and $\mathbf{C})$ mLMVECs were stimulated with increasing doses of IL-1 $\beta$ for 24 hours $(\mathbf{B})$ or stimulated with IL-1 $\beta(5 \mathrm{ng} / \mathrm{mL})$ over time (C). Protein expression was detected by Western blot. (D-C) $\mathrm{mLMVECs}$ were stimulated with IL-1 $\beta$ ( $5 \mathrm{ng} / \mathrm{mL}$ ) over time. RNA expression levels were analyzed by RT-PCR (D). Ubiquitination status of CREB was analyzed by immunoprecipitation with CREB antibody and blotting with an anti-ubiquitin antibody (E). SAC activity was detected $(\mathbf{F})(n=4)$. Intracellular CAMP levels were measured $(\mathbf{C})(n=4)$. (H) CREB itself binds to the putative CREs within the CREB promoter in $\mathrm{mLMVECs}$ by ChIP assays. The amplified region surrounds each proposed CRE binding element within the mouse CREB promoter

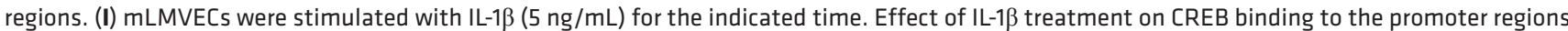
(CRE2 and CRE6) in mLMVECs was investigated by ChIP assays $(n=3) .{ }^{*} P<0.05 ;{ }^{*} P<0.01 ;{ }^{* * *} P<0.001$; NS, no significant difference. Statistics obtained from ANOVA. 
A

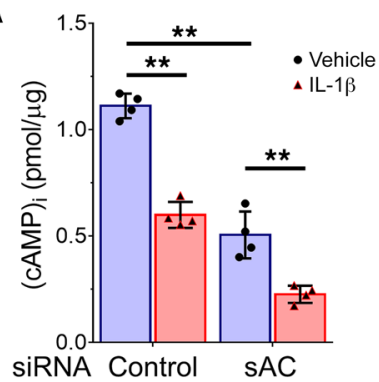

B

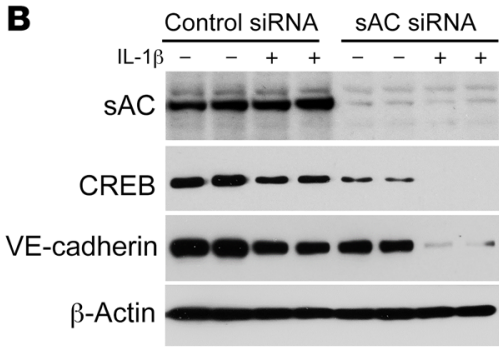

C

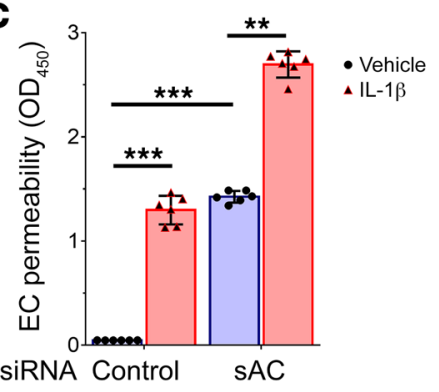

D

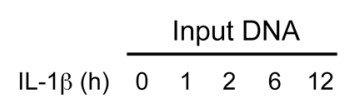

ChIP DNA
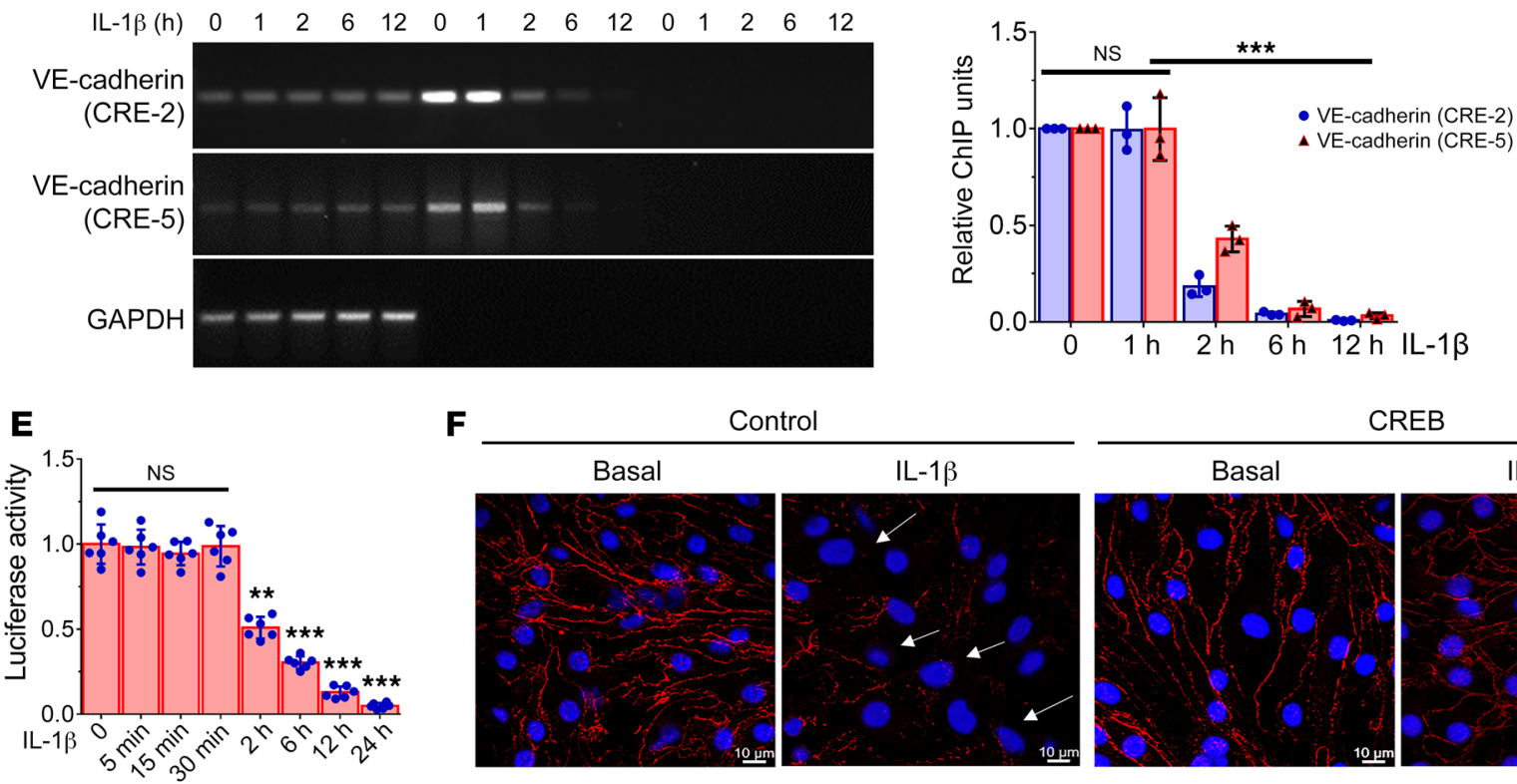

$\mathbf{F}$ Control CREB
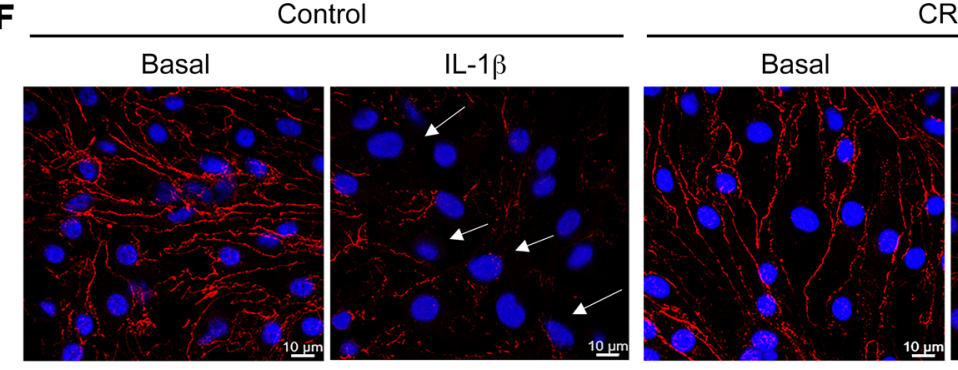
IL-1 $\beta$

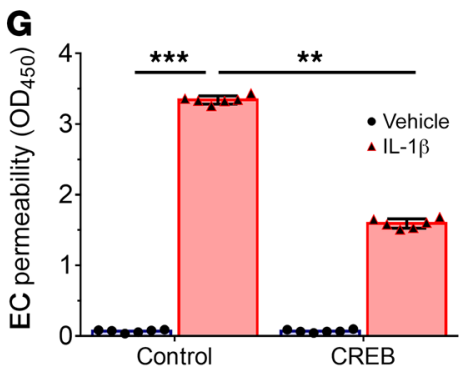

H

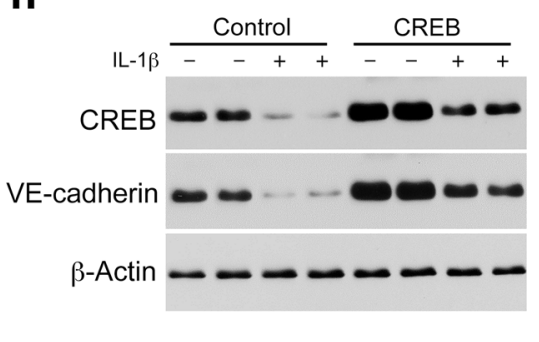

Figure 3. IL-1 $\beta$ transcriptionally downregulates CREB-mediated VE-cadherin expression to increase endothelial permeability. (A-C) mLMVECs were transfected with siRNA of sAC or control scrambled siRNA for 3 days. Cells were then stimulated with IL-1 $\beta$ ( $5 \mathrm{ng} / \mathrm{mL}$ ) for 12 hours. Intracellular cAMP levels were measured $(\mathbf{A})(n=4)$. Protein expression by Western blot $(\mathbf{B})$ and permeability to HRP $(\mathbf{C})$ were determined $(n=6)$. (D) mLMVECs were stimulated with IL-1 $\beta(5 \mathrm{ng} / \mathrm{mL})$ for the indicated time. Effect of IL-1 $1 \beta$ treatment on CREB binding to the mouse VE-cadherin promoter regions (CRE2 and CRE5) in mLMVECs was investigated by ChIP assays $(n=3)$. (E) mLMVECs were transiently transfected with the 2.5 -kb VE-cadherin promoter luciferase construct, CREB expression vector, and the internal control p-RL-TK vector by lipofectamine 3000 reagent for 2 days. Cells were then treated with IL-1 $\beta$ ( $5 \mathrm{ng} / \mathrm{mL}$ ) over time. CREB-regulated VE-cadherin transcription activity was measured using the dual luciferase reporter assay system $(n=6)$. (F-H) mLMVECs were transfected with CREB plasmids or control for 2 days, and stimulated with IL-1 $13(5 \mathrm{ng} / \mathrm{mL})$ for 1 day. Immunofluorescence staining of VE-cadherin (F); arrows denote disruption of endothelial barrier integrity. Permeability to $\operatorname{HRP}(n=6)(\mathbf{C})$ and protein expression by Western blot $(\mathbf{H})$ were determined. ${ }^{* *} P<0.01 ;{ }^{* *} P<0.001 ;$ NS, no significant difference. Statistics obtained from ANOVA. Scale bars: $10 \mu \mathrm{m}$.

As downregulation of CREB reduced VE-cadherin transcription in inflammatory lung injury (Figure 1), we surmised that ectopic expression of CREB in ECs would suppress the injury response. We observed that ectopic expression of $C R E B^{\mathrm{EC}}$ in control mice significantly reduced neutrophil infiltration (Figure 5, A and B) and overall leukocyte accumulation (Figure 5C) as well as lung vascular injury (Figure 5, D and $\mathrm{E}$ ) in response to LPS. These mice when subjected to a lethal dose of LPS ( $20 \mathrm{mg} / \mathrm{kg}$ i.p.) also showed $90 \%$ survival as compared with the control group showing only $10 \%$ survival (Figure $5 \mathrm{~F}$ ). Moreover, in the CLP polymi- 
A

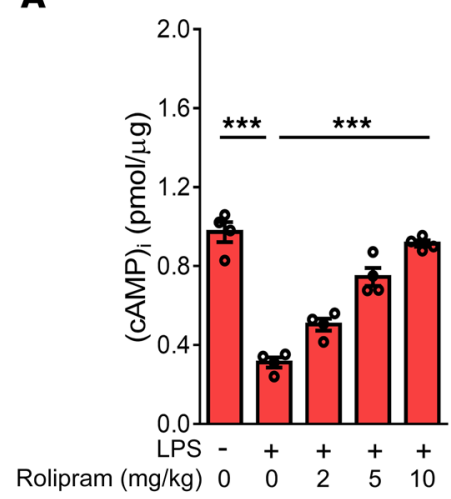

B

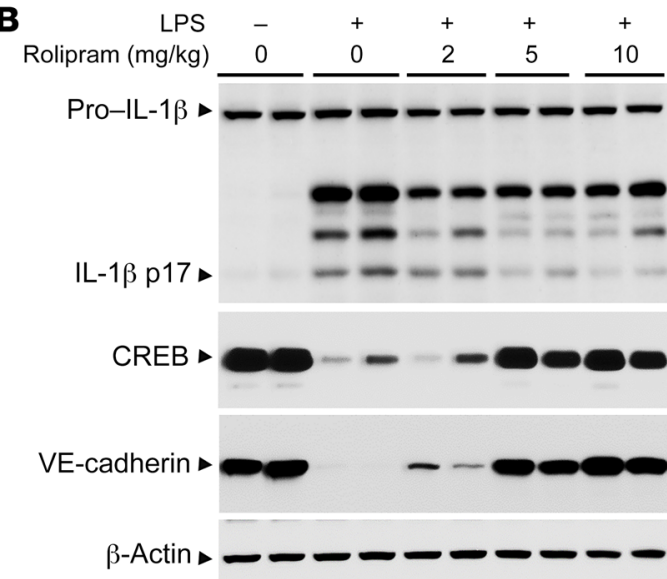

C

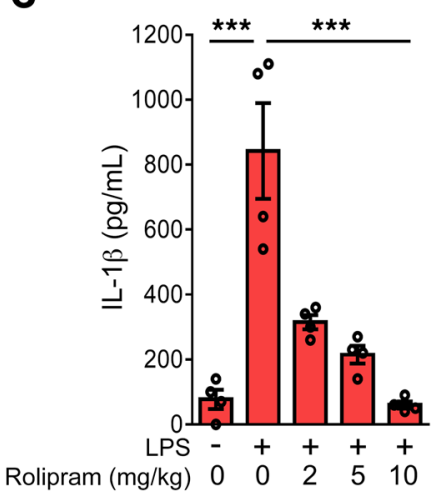

D

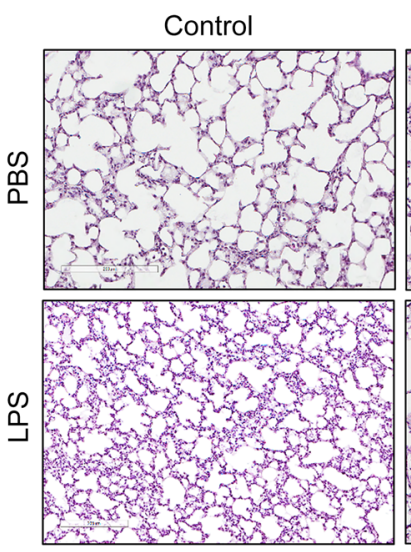

E

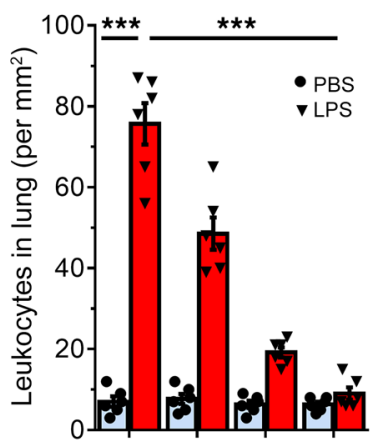

Rolipram (mg/kg) $0 \quad 2 \quad 5 \quad 10$
Rolipram (2 mg/kg)
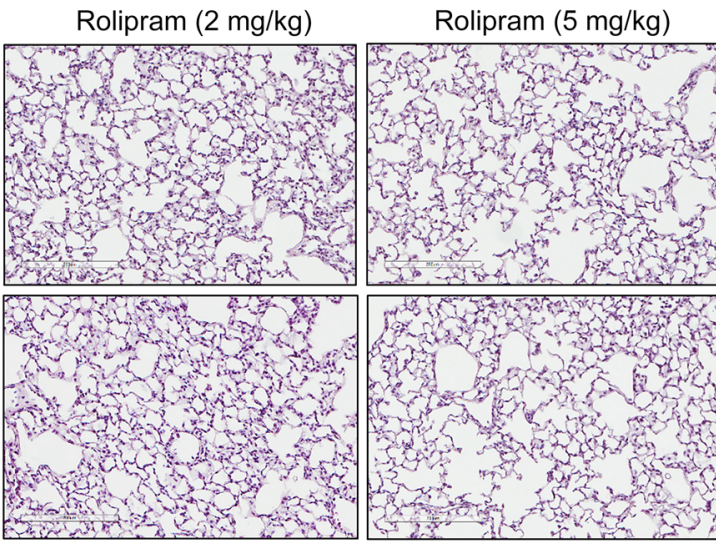

Rolipram (10 mg/kg)

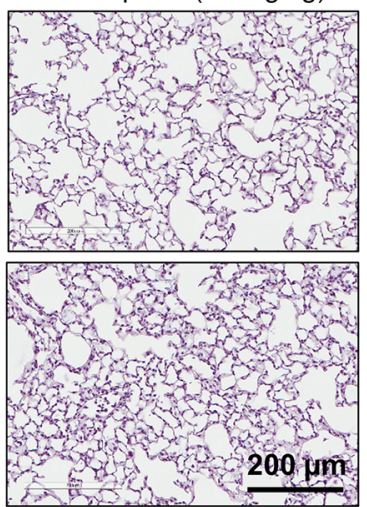

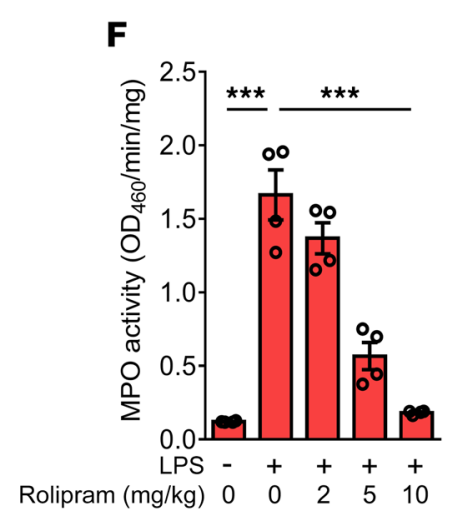

G

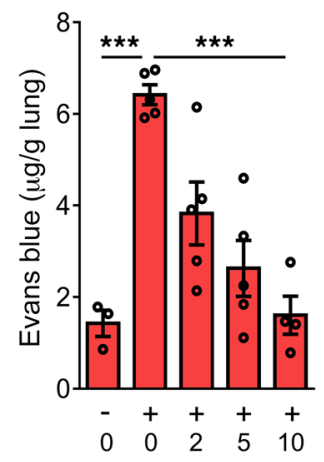

H

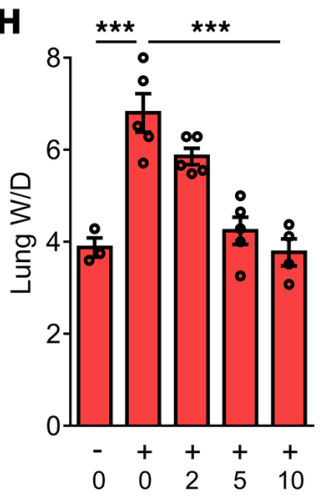

Figure 4. Augmentation of cAMP signaling by rolipram suppresses inflammatory lung injury. (A) C57BL/6) mice $(n=4)$ were administrated with rolipram $(2,5$, and $10 \mathrm{mg} / \mathrm{kg})$ for 1 hour, and injected intraperitoneally with LPS $(12 \mathrm{mg} / \mathrm{kg})$ for 1 day. Intracellular cAMP levels of the lung lysates were measured by ELISA. (B) Lung lysates were prepared for expression detection of IL-1 $\beta$ maturation, CREB, and VE-cadherin by Western blot. (C) Measurement of IL-1 $\beta$ levels in mouse serum, as determined by ELISA $(n=4)$. (D) H\&E staining of the lung is shown (scale bar: $200 \mu \mathrm{m} ; n=3$ ). (E) Quantitative analysis for leukocyte infiltration in lungs $(n=6)$. (F) Quantitative analysis of neutrophil infiltration by measurement of lung tissue MPO activity $(n=4)$. (G) Lung vascular permeability is detected by EBD leakage in the lungs $(n=3-5)$. Extracted dye contents in the formamide extracts are quantified by measuring at $620 \mathrm{~nm}$. (H) The ratios of the wet lung to dry lung weight were assessed $(n=3-5)$. Results are shown as mean \pm SEM. ${ }^{* *} P<0.001$. Statistics obtained from ANOVA.

crobial sepsis model of inflammatory lung injury, we observed that $C R E B^{\mathrm{EC}}$ mice displayed markedly reduced neutrophil infiltration (Figure 5, G and $\mathrm{H}$ ) and leukocyte accumulation (Figure 5I) as well as lung vascular injury (Figure 5J) and pulmonary edema (Figure $5 \mathrm{~K}$ ) when compared with controls. Furthermore, $C R E B^{\mathrm{EC}}$ mice showed significantly increased survival after CLP as compared controls (Figure $5 \mathrm{~L}$ ).
Endothelial cell-specific deletion of CREB promotes inflammatory lung injury. To address the requisite and sufficient role of endothelial CREB signaling in mediating lung vascular injury, we next generated endothelial-specific $C R E B$ knockout mice by backcrossing CREB floxed with Cdh5-Cre-ERT2 mice (Figure 6A). Loss of endothelial CREB (CREB $\left.B^{\mathrm{EC}-/}\right)$ in mice as compared with control mice induced basal reduction in VE-cadherin expression (Supple- 
A
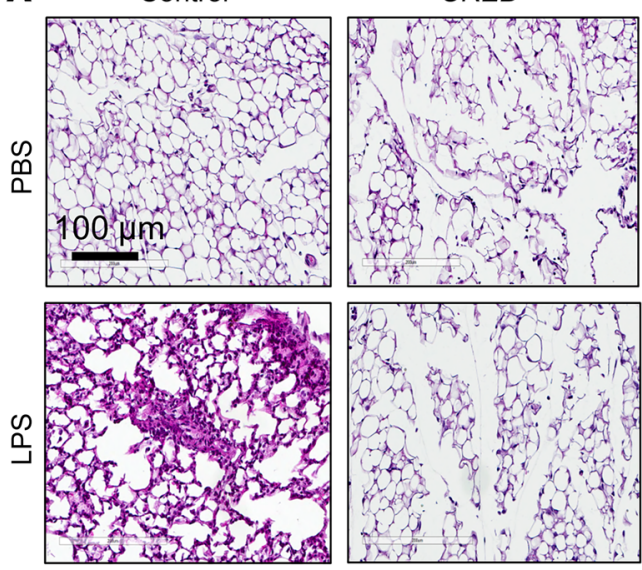

D

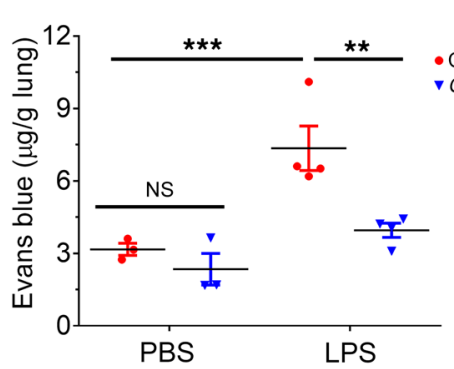

G
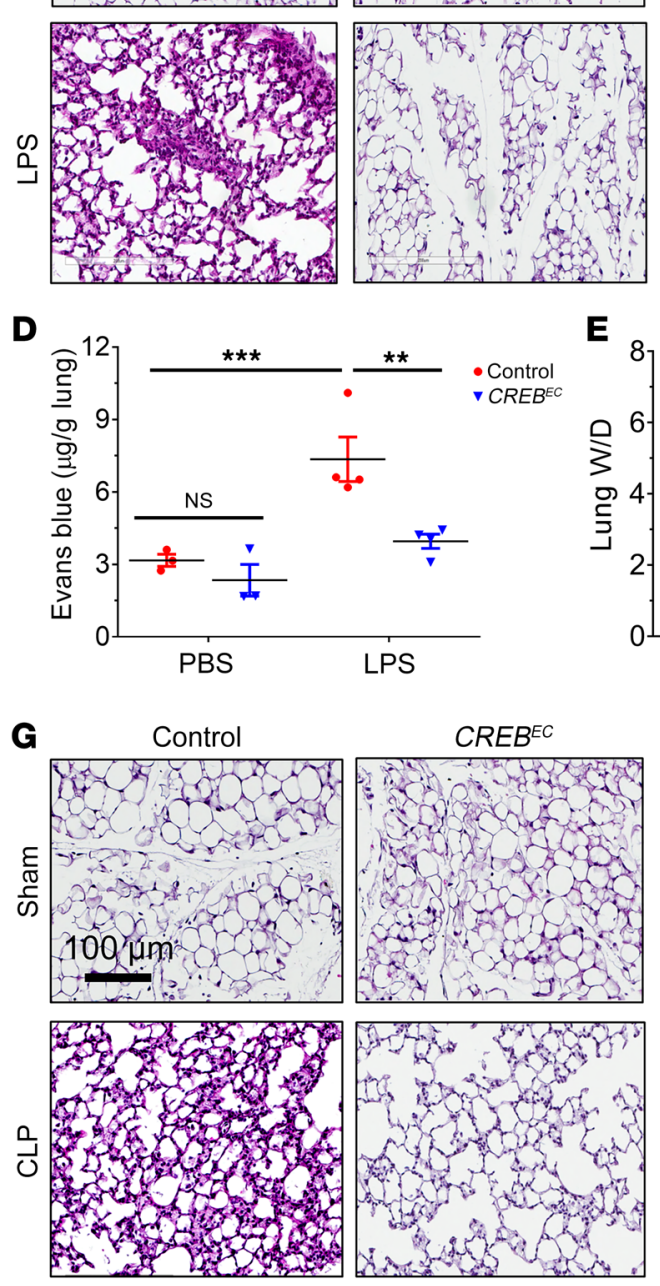

B

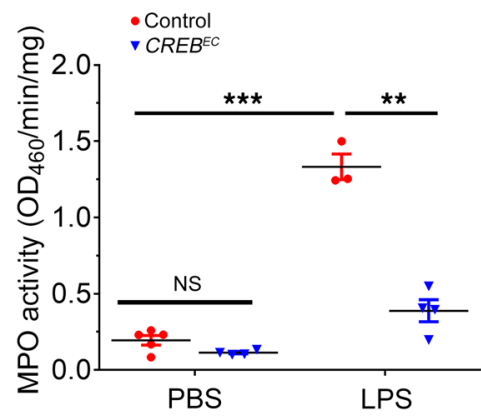

C

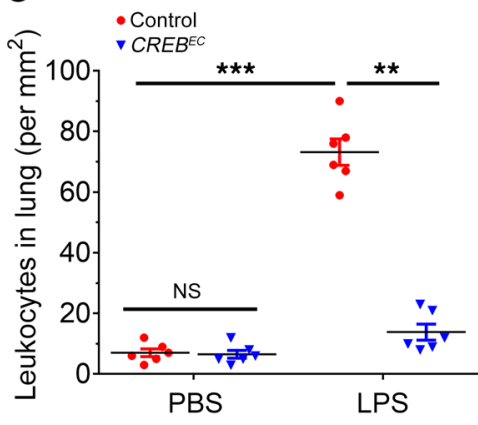

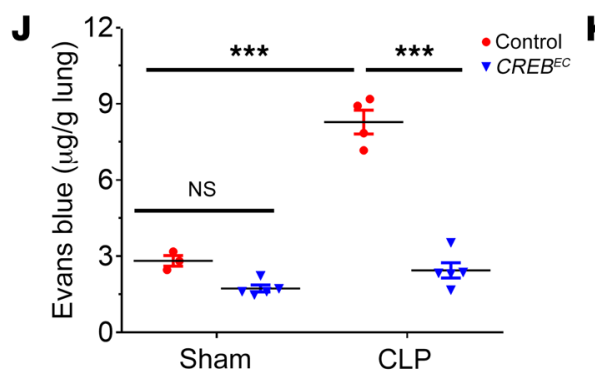
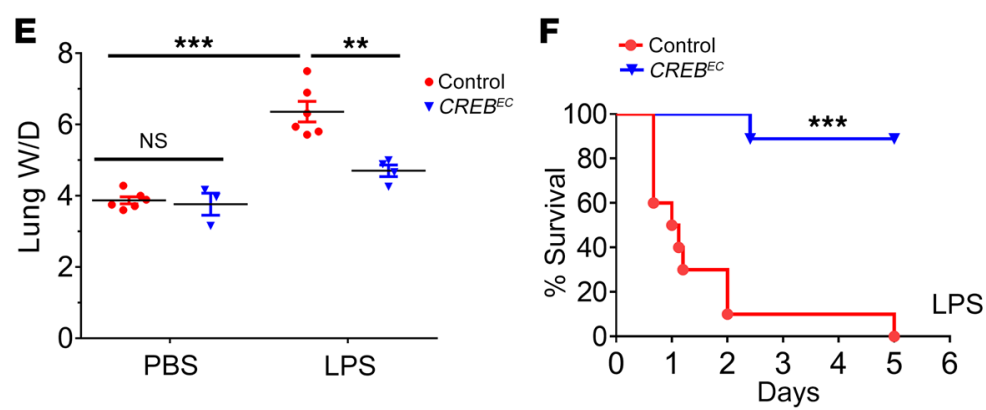

H
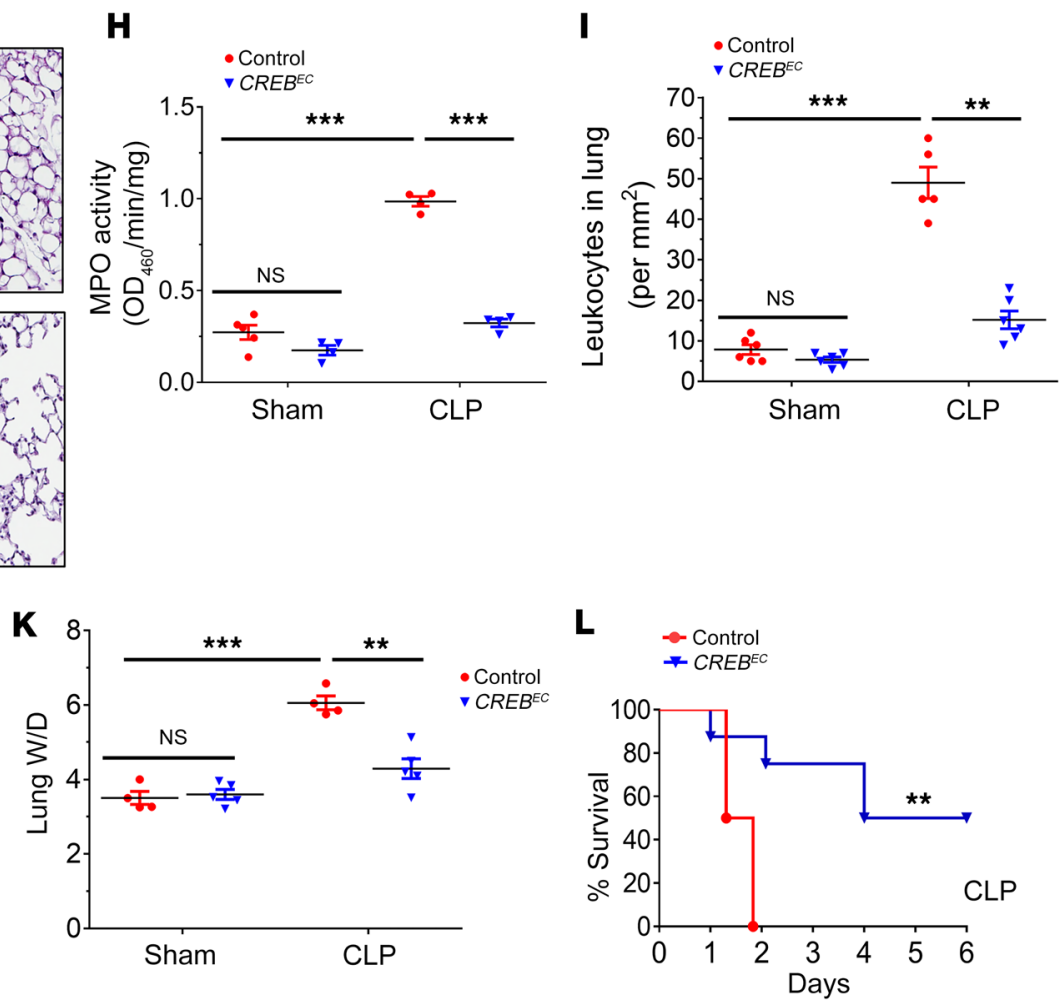
Figure 5. Ectopic expression of CREB in endothelial cells prevents inflammatory lung injury. (A-E) The 2.5-kb VE-cadherin promoterdirected CREB expression constructs (CREB ${ }^{\mathrm{EC}}$ ) and control plasmids were delivered into [57BL/6] mice by liposome-mediated retroorbital injection for 7 days. Mice were then injected intraperitoneally with LPS $(12 \mathrm{mg} / \mathrm{kg})$ for 1 day. (A) H\&E-stained cross-section of the lung from control and $C R E B^{\mathrm{EC}}$ gene-delivered mice shows inflammation and lung injury in control, but not in mice delivered by CREB ${ }^{\mathrm{EC}}$ (scale bar: $100 \mu \mathrm{m} ; n=3$ ). Quantitative analyses for lung tissue MPO activity (B) and leukocyte accumulation in lungs (C) were performed $(n=3-6)$. (D) Lung vascular permeability was detected by EBD leakage in the lungs isolated from control and $C R E B^{\mathrm{EC}}$ gene-delivered mice $(n=3-4)$. Extracted dye contents in the formamide extracts are quantified by measuring at $620 \mathrm{~nm}$. (E) The ratio of the wet lung to dry lung weight was assessed $(n=4-6)$. (F) Mice $(n=10)$ were delivered by control and CREB ${ }^{\mathrm{EC}}$ for 7 days. Survival of mice subjected to a lethal dose of LPS $(20 \mathrm{mg} / \mathrm{kg}$ i.p.) for the indicated days was monitored and is presented as a Kaplan-Meier plot. (G-K) Mice were delivered by control and $C R E B^{\mathrm{EC}}$ for 7 days, and then subjected to CLP surgery for 1 day. (C) Representative H\&E staining of lung sections from control and $C R E B^{\mathrm{EC}}$ gene-delivered mice is shown (scale bar: $100 \mu \mathrm{m}$; $n=3)$. (H) Quantitative analyses for lung tissue MPO activity $(n=4-5)$ and (I) leukocyte accumulation in lungs were measured $(n=5-6)$. (J) Lung vascular permeability was detected by EBD leakage $(n=3-5)$. (K) The ratio of the wet lung to dry lung weight was determined $(n=4-5)$. (L) Survival of mice after CLP surgery for the indicated days was monitored and is presented as a Kaplan-Meier plot $(n=10) .{ }^{* *} P<0.01 ;{ }^{* *} P<0.001$; NS, no significant difference. Statistics obtained from ANOVA.

mental Figure 8A), a moderate degree of lung inflammation as evidenced by increased MPO activity (Supplemental Figure 8B), generation of IL-1 $\beta$ (Supplemental Figure $8 \mathrm{C}$ ), small increase in basal lung vascular permeability (Supplemental Figure 8, D and E), and reduced survival rate in response to a lethal dose of LPS challenge (Supplemental Figure 8F). To investigate the effects of endothelialspecific loss of CREB on PMN dynamics in lungs, we used the intravital imaging approach (Supplemental Video 2). Deletion of endothelial-expressed CREB significantly increased PMN numbers and prolonged transit times and decreased PMN velocity (Supplemental Figure 9, A-E). We also observed enhanced adhesion of PMN to the endothelium in lungs of $C R E B^{\mathrm{EC}-/}$ mice in association with increased expression of adhesive proteins ICAM-1, LFA-1, and Mac-1 (Supplemental Figure 9F).

We next addressed whether restoration of CREB-regulated VE-cadherin signaling would reverse the lung inflammation and vascular injury seen in CREB $B^{\mathrm{EC}-/}$ mice. Employing the $2.5-\mathrm{kb}$ murine VE-cadherin promoter-directed CREB expression construct by nanoparticle liposome-mediated delivery as above, we observed that CREB-VE-cadherin signaling was successfully restored in endothelial cells isolated from $C R E B^{\mathrm{EC}-/}$ mouse lungs (Figure 6B). Notably, the development of lung inflammation in $C R E B^{\mathrm{EC}-/}$ mice, as evaluated by neutrophil infiltration by H\&E staining (Figure 6C), leukocyte accumulation (Figure 6D), increased MPO activity (Figure 6E), and production of proinflammatory cytokines, including IL-1 $\beta$ (Figure 6F), was reversed in endothelial CREB-restored mice. Furthermore, restoration of CREB-VE-cadherin signaling in endothelial cell-specific CREB-deficient mice restored lung vascular permeability (Figure 6G) and fluid balance (Figure 6H).

We also evaluated the effects of rolipram on LPS-induced lung vascular injury in $C R E B^{\mathrm{EC}-}-\mathrm{m}$ mice. Rolipram treatment of mice after LPS challenge showed significantly reduced lung vas- cular injury (Figure 7, A-C). This protective effect was abolished in $C R E B^{\mathrm{EC}-/}$ mice, suggesting that activation of the endothelial CREB-VE-cadherin axis is a potential therapeutic target preventing endotoxemia-induced lung vascular injury. To evaluate whether sepsis-induced IL-1 $1 \beta$ production and reduced cAMPCREB-VE-cadherin signaling cascade are clinically relevant, we measured cAMP, IL-1 $\beta$, and IL-1 receptor antagonist (IL-1RA) levels in plasma from patients with ARDS and cardiogenic pulmonary edema (CPE). ARDS but not CPE patients exhibited augmented levels of IL-1 $\beta$ (Figure 7D) and IL-1RA (Figure 7E) as well as decreased plasma cAMP concentrations (Figure 7F) as compared with healthy controls. There was no significant difference between CPE patients and healthy controls.

\section{Discussion}

Lung vascular leakage in response to an unchecked cytokine storm generated by activation of innate immune cells such as the production of IL-1 $\beta$ from proinflammatory macrophages is a hallmark of sepsis-induced inflammatory injury $(25,26)$. The vascular leakage in lungs is the result of endothelial barrier breakdown at the level of AJs that control the flux of liquid and protein across the barrier $(27,28)$. Endothelial permeability is normally finely tuned by homotypic interaction of VE-cadherin in endothelial monolayers, a central component of AJs, as well as associated catenins interacting with VE-cadherin and actin cytoskeleton $(12,13)$. Although multiple studies have demonstrated that posttranslational modifications of VE-cadherin are important for regulation of VE-cadherin junctions $(12,14)$, precious little is known about transcriptional regulation of VE-cadherin expression and its role in mediating inflammatory lung injury. Here, we showed that the transcriptional factor CREB regulated VE-cadherin expression through direct promoter binding, and downregulation of CREB-mediated VE-cadherin expression in endothelial cells was a central event in mediating sepsis-induced inflammatory lung injury.

IL-1 $\beta$ is a key mediator in sepsis-induced inflammation and tissue injury (10, 11, 29). Macrophage-generated IL-1 $\beta$ acting through IL-1R1 during sepsis disrupted vascular stability $(11,26)$. Pharmacological approaches used to target IL-1 $\beta$ signaling including IL-1R antagonists, soluble decoy receptors, and human IL-1 $\beta$ monoclonal antibody (canakinumab), have been recently tested $(30,31)$. Targeting the IL-1 $\beta$ pathway ameliorated atherosclerosis in patients (30), although their effectiveness in ARDS patients has not been completely addressed. As IL-1 $\beta$ has a key role in host defense, its suppression may lead to increased infection and sepsis-related death (32). Thus, delineating the mechanisms by which IL-1 $\beta$ disrupts lung endothelial permeability would allow for the development of targeted therapies without compromising the lung's host-defense functions. Here, we showed that IL-1 $1 \beta-$ induced downregulation of the CAMP-CREB-VE-cadherin pathway was a key mechanism underlying sepsis-induced lung endothelial barrier impairment and lung injury.

Clinical benefits of IL- $1 \beta$ signaling inhibitors such as recombinant human IL-1R antagonist for sepsis-related respiratory diseases such as ARDS have been equivocal (33-35). SNP studies showed that expression of IL-1R antagonist in patients (SNP rs315952C) was associated with decreased risk of ARDS (34), suggesting that IL-1R antagonism may attenuate ARDS risk. We observed elevat- 

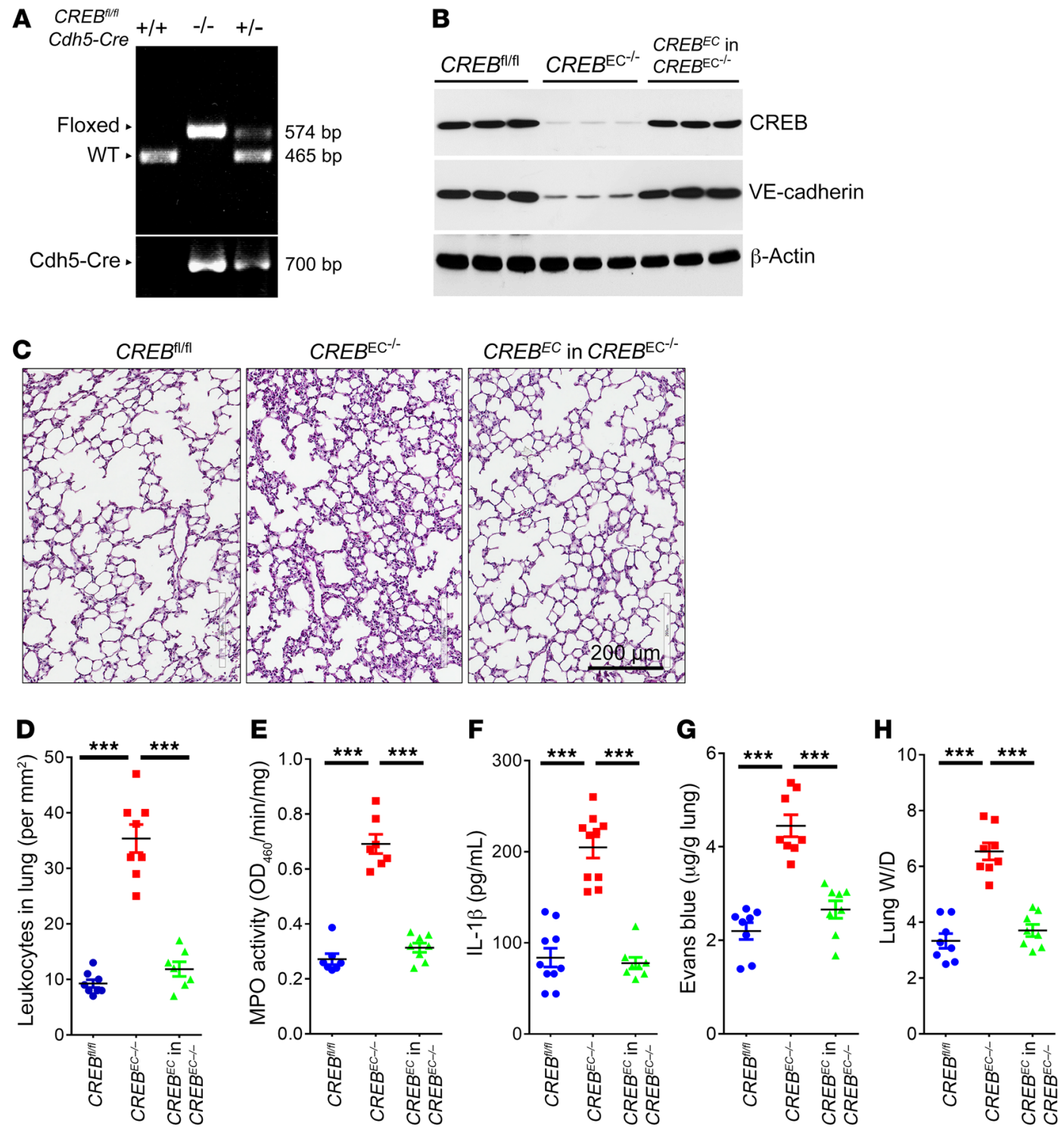

Figure 6. Endothelial CREB expression is required and sufficient for inflammatory lung injury in mice. (A) EC-specific CREB knockout mice were generated by backcrossing $C R E B^{\mathrm{fl} / \mathrm{fl}}$ mice with $C d h 5-C r e-E R T$ mice. (B) The 2.5 -kb VE-cadherin promoter-directed CREB expression constructs were delivered into CREB ${ }^{\mathrm{EC}-I-}$ mice $(n=3)$ by liposome-mediated retroorbital injection for 7 days. Endothelial cells were isolated from mice using anti-CD31 beads. EC-specific

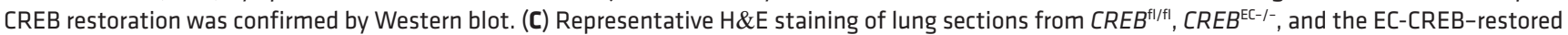
CREB ${ }^{\mathrm{EC}-1-}$ mice (scale bar: $200 \mu \mathrm{m} ; n=3$ ). (D) Quantitative analysis for leukocyte infiltration in lungs $(n=7)$. (E) Measurement of lung tissue MPO activity $(n=7)$. (F) Inflammatory cytokine levels of IL-1 $\beta$ in mice serum were measured by ELISA $(n=7-10)$. (G) Lung vascular permeability is detected in lungs from $C R E B^{\mathrm{fl} / \mathrm{fl}}, C R E B^{\mathrm{EC}-1-}$, and the EC-CREB-restored CREB ${ }^{\mathrm{EC}-/-}$ mice $(n=8)$. (H) The ratio of the wet lung to dry lung weight was determined $(n=8)$. ${ }^{* *} P<0.001$. Statistics obtained from 1-way ANOVA.

ed plasma concentrations of IL-1 $\beta$ and IL-1RA in ARDS patients as compared with healthy controls, findings consistent with previous reports $(34,35)$. Although measurements of individual cytokines in serum and bronchoalveolar lavage (BAL) of ARDS patients did not predict the onset or outcome of $\operatorname{ARDS}(34,36)$, elevated circulating plasma concentration of IL-1R antagonists in ARDS patients is a possible measure of outcome and may inform the value of targeting IL-IR. Meyer et al. showed that blockade of IL-1 $\beta$ signaling by IL-1RA exhibited significant survival benefits in sepsis patient subgroups (preenrollment IL1RA concentrations greater than 2071 pg/ $\mathrm{mL}$ ), but not in subjects with a concentration less than $2071 \mathrm{pg} / \mathrm{mL}$
(37). The plasma IL-1RA levels in our tested ARDS patients were greater than this cut-off point. Thus, the efficacy of IL-1RA treatment at least in subgroups of sepsis patients holds promise.

The conclusion that IL- $1 \beta$-induced downregulation of the cAMP-CREB-VE-cadherin axis is a key mechanism underlying sepsis-induced lung endothelial barrier impairment and lung injury was based on multiple lines of evidence. We employed a combination of global and endothelial-specific genetic knockout mouse models, 2 independent endotoxemia-LPS and polymicrobial infection CLP sepsis models, and liposomal nanoparticledirected, endothelial-specific gene delivery in the lung endothe- 

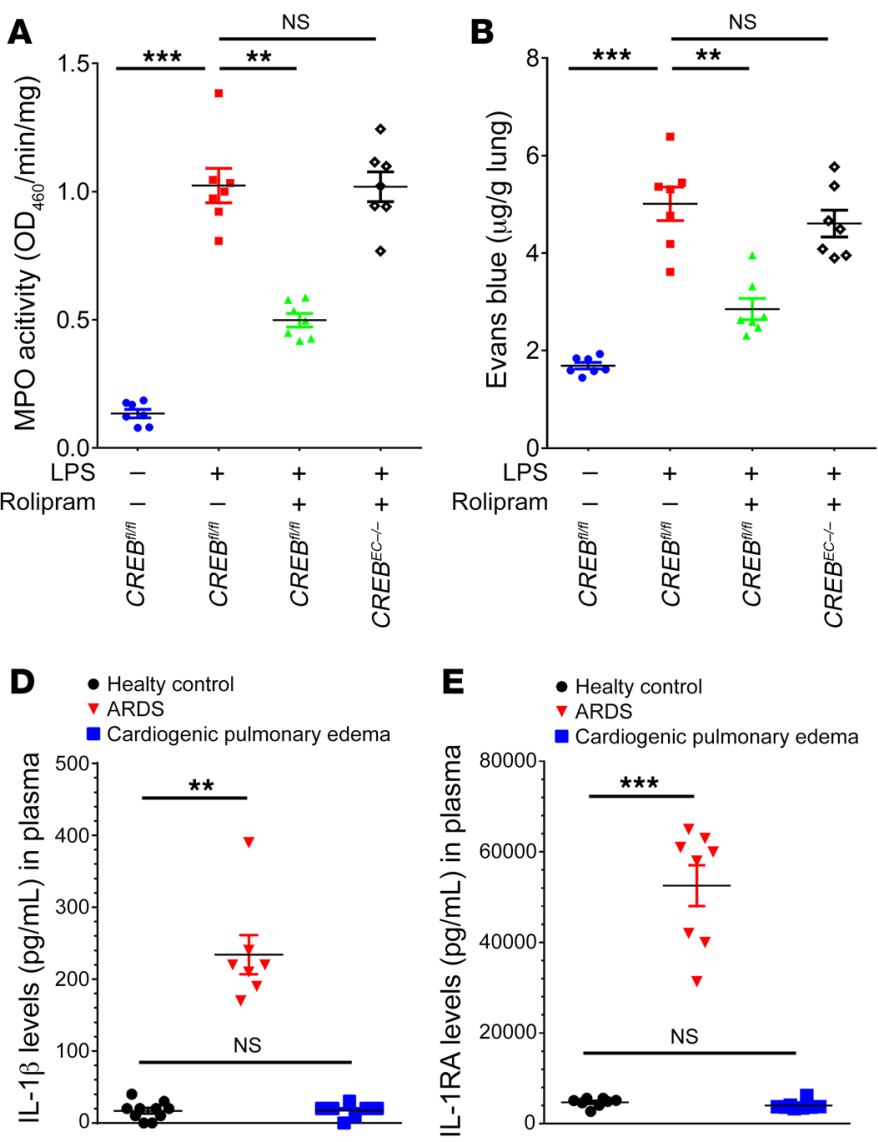

lium to demonstrate the essential role of cAMP-CREB-VE-cadherin pathway in mediating IL-1 $\beta$-induced lung endothelial injury. We showed that IL-1 $\beta$ via binding to receptor IL-1R1 in endothelial cells reduced the activity of SAC, resulting in downregulation of cAMP generation and expression of the transcription factor CREB, and thereby reduced the expression of VE-cadherin and promoted loss of VE-cadherin junctional integrity. The CREB-dependent loss of VE-cadherin appeared to be the primary mechanism of vascular injury since targeted restoration of CREB expression in lung endothelial cells reversed sepsis-induced inflammatory lung injury (as described in Figure 6). Notably, the cAMP-CREB pathway of lung injury was also amenable to pharmacological therapy. We used rolipram, a selective inhibitor of PDE4. Rolipram prevents hydrolysis of cAMP and maintains high cellular cAMP concentrations (20). Preventing LPS-induced reduction in cAMP concentration in this manner abrogated IL-1 $\beta$-induced reduction in CREB activity and VE-cadherin expression.

Pyroptosis is induced in endothelial cells by the binding of intracellular LPS to caspase-11, which results in cleavage of IL-1 $\beta$ and membrane pore-forming protein Gasdermin D (Gsdmd) (8). Gsdmd pores function as a conduit for secretion of proinflammatory IL-1 $\beta$ into the circulation (38). We showed the essential role of intracellular LPS-sensing caspase-11 in mediating endothelial pyroptosis and IL-1 $\beta$ production during sepsis-induced acute lung injury (ALI) (8). However, the mechanistic link between endothelial pyroptosis and disruption of endothelial barrier AJs remains unknown. It appears that only a small pool of endothelial cells undergoes pyroptosis after LPS (8). Presumably, this is related

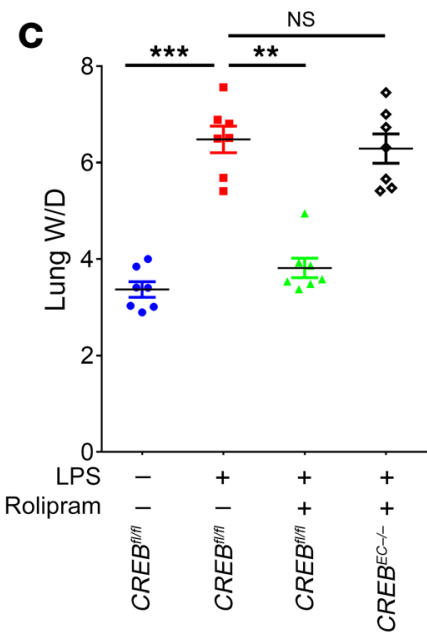

Figure 7. CREB-VE-cadherin axis is required for ameliorative effects of rolipram on LPSinduced lung vascular injury. (A-C) CREB ${ }^{\mathrm{fl} / \mathrm{fl}}$ and $C R E B^{\mathrm{EC}-I-}$ mice $(n=7)$ were injected i.p. with LPS $(8 \mathrm{mg} / \mathrm{kg}$ ) for 1 day, and administrated with rolipram $(5 \mathrm{mg} / \mathrm{kg})$ or control vehicle for another day. (A) Measurement of lung tissue MPO activity. (B) Changes in lung vascular permeability under different conditions. (C) The ratio of wet lung to dry lung weight measurements to assess pulmonary edema. (D-F) Circulating concentrations of IL-1B (D), IL-1 receptor antagonist (IL-1RA) (E), and CAMP (F) in plasma of healthy volunteers (control) and ALI/ARDS and CPE patients $(n=7-10)$ were measured by ELISA. ${ }^{* *} P<0.01$; ${ }^{* *} P<0.001$; NS, no significant difference by 1-way ANOVA.

to activation of the membrane repair mechanisms such as the endosomal sorting complex required for transport (ESCRT) pathway (39). The present study implies that IL-1 $\beta$ releases from the pyroptotic immune cell pool, macrophages, and endothelial cells, and targets nonpyroptotic endothelial cells via repression of the cAMP-CREB-VE-cadherin signaling cascade to disrupt endothelial barrier stability.

Zhu et al. (11) showed that IL-1 $\beta$ disrupted VE-cadherin cellsurface localization to impair endothelial stability in a human in vitro cell model via an NF- $\kappa \mathrm{B}$-independent pathway that relied on signaling through the small GTPase ADP-ribosylation factor 6 (ARF6) and its activator ARF nucleotide binding site opener (ARNO). As ARNO bound the adaptor protein MYD88, the MYD88-ARNO-ARF6 pathway mediated IL-1 $\beta$-induced endothelial disruption (11). However, it is not known how or if the MYD88ARNO-ARF6 pathway intersects with the cAMP-CREB-VE-cadherin pathway described herein. It is likely that a cytokine as pleiotropic as IL-1 $\beta$ injures the endothelium by multiple pathways that depend on concentrations, duration of exposure, and types of endothelial cells activated. Zhu et al. (11) used a higher dosage of IL-1 $\beta(10 \mathrm{ng} / \mathrm{mL})$ in contrast to the present study $(2-5 \mathrm{ng} / \mathrm{mL})$. We demonstrated that the drug rolipram, a cell-permeable selective inhibitor of PDE4 (which mediates cAMP degradation), as well as multiple endothelial-specific genetic approaches, prevented sepsis-induced loss of lung endothelial integrity and markedly improved outcomes in animal model of inflammatory lung injury.

The observed lung endothelial barrier protective role of cAMP-CREB signaling hearkens back to endothelial barrier pro- 
tection elicited acutely by raising endothelial cell concentration of cAMP (40). In these studies, cAMP restored endothelial permeability in response to multiple permeability-increasing stimuli such as thrombin (40) and ischemia/reperfusion (41). The barrier protection was ascribed to cAMP-dependent activation of guanine nucleotide exchange factor (EPAC), which promoted exchange of GTP in the small GTPase Rap1 $(40,42)$. Rap1 enhanced reduced endothelial permeability by promoting AJ assembly (42). In the present study, however, the endothelial barrier effect of cAMPCREB signaling was due to transcriptional control of VE-cadherin expression and led to repair of the endothelium. The transcriptional regulation of endothelial permeability required hours, in contrast to the rapid response elicited by cAMP; these 2 cAMPmediated mechanisms appear to be independent.

sAC (ADCY10) generates cAMP pools within various cellular compartments; cytosol, mitochondria, nucleus, and subplasmalemma space (43). Studies showed that cAMP compartmentalization, depending on its localization, may exert opposite effects on endothelial permeability (44-47). The effects of cAMP signaling are thus highly complex and dynamic (48). The distinct roles of compartmentalized cAMP signaling also depends on the specific AC isoforms coupled to specific GPCRs with different localization patterns and cAMP regulatory enzymes such as PDEs (48). Our evidence supports the concept that the intracellular cAMP signaling pool generated by SAC and downstream CREB activation was endothelial barrier protective. The present study shows CREB directly regulates VE-cadherin expression. We demonstrated that depletion of SAC in endothelial cells reduced CAMP generation and the expression of CREB and VE-cadherin, and increased endothelial permeability. These results together support the role of IL-1 $\beta$ in inhibiting CAMP-CREB-VE-cadherin signaling and thereby increasing endothelial permeability.

An important question is how LPS-generated IL-1 $\beta$ impairs SAC activity to reduce intracellular cAMP levels resulting in defective CREB induced expression of VE-cadherin. The intracellular concentrations of cAMP are tightly controlled by the activity of sAC, which synthesizes cAMP from ATP, and the cyclic nucleotide PDEs, which hydrolyze cAMP (21). Our data showed that IL-1 $\beta$ reduced SAC activity and CAMP concentrations within a few minutes, ruling out the possibility that reduction in CAMP was due to induction of PDE expression. Sepsis is known to severely disrupt the acid-base, ion, and $\mathrm{pH}$ status of cells (49). Based on the crystal structure of human SAC catalytic domains, it was shown that an anionic inhibitor, 4,4'-diisothiocyanatostilbene-2,2' -disulfonic acid (DIDS), prevented sAC activation through binding to the active site entrance and inhibited SAC by steric hindrance (50). Thus, it is possible that a similar perturbation through generation of anionic inhibitors interferes with sAC in a disease as complex as sepsis, resulting in defective CREB expression and CREB transcription of $V E$-cadherin.

Although cAMP-CREB is known to control the expression of multiple genes via binding to CREs within promoter regions (51), we focused on CAMP-CREB signaling in regulating the endothelial barrier via VE-cadherin expression. We identified for the first time VE-cadherin as the crucial target of endothelial CREB during sepsis-induced lung vascular leakage. We cannot exclude the possibility that other transcriptional factors HIFs and SOX17, known to be involved in regulating the integrity of AJs via expression of VE-cadherin $(17,24)$, can also participate in a homeostatic process as fundamental as maintenance of endothelial barrier function. In summary, the present study provides what we believe are novel insights into the mechanism of IL-1 $\beta$-induced inflammatory lung injury. IL-1 $\beta$ deactivated cAMP-CREB signaling and suppressed VE-cadherin expression in lung endothelial cells, raising the prospect of preventing inflammatory lung injury by selectively interfering with the cAMP-CREB pathway.

\section{Methods}

Mice. C57BL/6 mice were purchased from Charles River Laboratory. $I L-1 R 1^{-/-}$and $V E$-cadherin (Cdh5)-Cre-ERT2 mice were purchased from Jackson Laboratory. Endothelial-specific $C R E B^{-/-}$mice were generated by backcrossing $C R E B$ floxed and $C d h 5$-Cre-ERT 2 mice. Genotyping of mice was performed by PCR using tail DNA. The seventh and eighth generations of the backcrossed offspring were used for studies. All experimental mice were 2 to 3 months old. For the number of animals needed to achieve statistically significant results, we conducted a priori power analysis. We calculated power and sample sizes according to data from small pilot experiments, variations within each group of data, and variance similarities between the groups that were statistically compared. Animals with sex- and age-matched littermates were randomly included in experiments. No animals had to be excluded because of illness after experiments. Animal experiments were carried out in a blinded fashion.

Cell preparation and culture. Murine and human lung microvascular endothelial cells (mLMVECs and hLMVECs) were isolated and cultured as previously described (8). In brief, mouse lungs were minced, digested with collagenase at $37^{\circ} \mathrm{C}$ for 60 minutes, and centrifuged at $1000 \mathrm{~g}$. Cell suspension was incubated with anti-CD31-coated Dynabeads for 2 hours, and ECs were magnetically sorted. Isolated ECs were cultured with DMEM containing endothelial growth supplement.

Antibodies and reagents. We purchased antibodies targeting IL-1 $\beta$ (R\&D Systems, AF-401-NA), soluble adenylate cyclase 10 (Aviva Systems Biology, ARP47447_P050), CREB (Cell Signaling, 48H2), p-CREB Ser133 (Cell Signaling, 9198), Ubiquitin (Invitrogen, 13-1600), VE-cadherin (Santa Cruz Biotechnology, sc-6458), ICAM-1 (Santa Cruz Biotechnology, sc-1511), LFA-1 (Santa Cruz Biotechnology, sc-15327), Mac-1 (Santa Cruz Biotechnology, sc-6614), NOS-3 (H-159) (Santa Cruz Biotechnology, sc-8311), p-eNOS (Ser114) (MilliporeSigma, 07-357), CD31 (Millipore, CBL13371), CD11B (BD Pharmingen, 553307), Flag (MilliporeSigma, F1804), $\beta$-catenin (MilliporeSigma, SAB4300470), GAPDH (Cell Signaling, 5174), and $\beta$-actin (Santa Cruz Biotechnology, sc-47778).

LPS-EB Ultrapure (catalog tlrl-3pelps) for in vitro study was purchased from InvivoGen. LPS (catalog L2630) for mice experiments, albumin (catalog A7906), O-Dianisidine dyhydrochloride (catalog D3252), 3,3',5,5'-tetramethylbenzidine (TMB, catalog T0440), and protease inhibitor cocktail (catalog P8340) were from MilliporeSigma. The 6.5-mm Transwell inserts (catalog 3413) were from Corning. Recombinant murine TNF- $\alpha$ (catalog 315-01A) was from PeproTech. Mouse IL-1 $\beta$ (catalog 14-8012-62) recombinant protein was from Thermo Fisher Scientific. cAMP direct immunoassay kit (catalog K-371-100) were from BioVision. Dual-Luciferase Reporter Assay System (catalog E1910) was from Promega. Mouse IL-1ß/IL-1F2 Quantikine ELISA Kit (catalog SMLBO0C), human IL-1ß/IL-1F2 Quantikine 
ELISA Kit (catalog DLB50), human IL-1RA/IL-1F3 Quantikine ELISA Kit (catalog DRA00B), and streptavidin-HRP (catalog DY998) were purchased from R\&D Systems. Enhanced chemiluminescence (ECL) Western blotting detection reagents and nitrocellulose membranes (Hybond-ECL) were from Amersham Biosciences. Lipofectamine 3000 transfection reagents were from Invitrogen. Basic nucleofector kit (catalog VPI-1001) for primary endothelial cells was from Lonza.

Construction of plasmids. Mouse CREB coding sequences (NM 001037726.1) were cloned into pcDNA3.0 expression vector at EcoRI and XhoI restriction enzyme sites with a flag tag using the primers: forward primer, ATAAGAATTCGACTACAAAGACGATGACGACAAGATGACCATGGAATCTGGAGCAG; reverse primer, ATAACTCGAGTTAATCTGATTTGTGGCA.

For cloning of mouse VE-cadherin (NM_009868) promoter regions, genomic DNA from mLMVECs or lung tissues was prepared using PureLink Genomic DNA mini kit (K1820-00, Invitrogen) following the manufacturer's instructions. The 1.3-, 2.0-, and $2.5-\mathrm{kb}$ murine $V E$-cadherin promoter regions were cloned into the upstream of CREB coding sequences at NdeI and EcoRI sites of expression plasmids using the following forward primers for the VE-cadherin promoters: ACTCGCATATGAAGCACACATATTCAG (2.5-kb); ACTCGCATATGAGGGTTGCTGTCCATCGTTTA (2.0-kb); ACTCGCATATGAGTATTTTCTCCATGCCCGGAA (1.3-kb). The common reverse primer was CGACTAGAATTCCGTGAGCAGAAGGCTCTCTCCA. For cloning of murine $V E$-cadherin luciferase reporter constructs, various fragments of the $5^{\prime}$ flanking sequence of the mouse $V E$-cadherin gene were amplified directly from mLMVECs genomic DNA by polymerase chain reaction (PCR) and subcloned into the pGL3-Basic vector (Promega) at the SacI and XhoI sites. The plasmids contain the regions from +18 to various sizes of the 5 -flanking region of the murine $V E$-cadherin gene with respect to the transcriptional start site. All PCR reactions were performed using the Expand High Fidelity PCR system (Roche Applied Science). PCR primers are described in Supplemental Table 2. Sequences of the constructs were determined by autosequencing to verify transcription in frame.

Luciferase reporter gene assay. mLMVECs in 24-well plates were cotransfected with VE-cadherin luciferase constructs or empty pGL3 vector (400 ng), expression vector encoding CREB or empty pcDNA3 vector (400 ng) and the internal control p-RL-TK vector (50 ng) using the Basic Nucleofector Amaxa transfection kit for 48 hours. Total amounts of DNA for each well were normalized by adding empty vector pcDNA3. Cells were harvested and luciferase activity was measured using the Dual-Luciferase Reporter Assay System (Promega, E1910) according to the manufacturer's instructions. The relative activity was normalized by the ratio of firefly luciferase activity to renilla luciferase activity (internal control) and calculated as the folds to control pGL3-basic or pcDNA3 empty vector. Data are from 2 to 3 independent experiments.

ChIP assay. ChIP assay was performed following the manufacturer's instructions (Upstate). mLMVECs were fixed with $1 \%$ formaldehyde at $37^{\circ} \mathrm{C}$ for 10 minutes, lysed, and sonicated. Soluble chromatin was coimmunoprecipitated with CREB antibodies or an equal amount of rabbit IgG. Cells at approximately $80 \%$ confluence grown in one $140-\mathrm{mm}$ diameter dish were used per immunoprecipitation. After decross-linking of the DNAs, DNA purified from starting (1\% input) and immunoprecipitated samples were subjected to PCR using the primers listed in Supplemental Tables 3 and 4. Those regions of amplification contain the potential CREB-binding CREs in the murine
VE-cadherin and CREB promoter regions. Standard PCR reactions were performed. The GAPDH promoter was analyzed by ChIP in parallel as a control. The relative ChIP units were calculated by the ratio of ChIP DNA to input DNA using densitometry.

mRNA expression analysis by RT-PCR and quantitative real-time $P C R$. One-step RT-PCR amplification was performed using the SuperScript 1-step RT-PCR system with Platinum Taq DNA polymerase (Invitrogen). One microgram total RNA isolated from IL-1 $\beta$-treated mLMVECs was used as a template for subsequent 1-step RT-PCR. GAPDH served as an internal control of the reaction. One-step RT-PCR products were analyzed by electrophoresis on $1.2 \%$ agarose gels containing ethidium bromide. For quantitative real-time PCR, total RNA was extracted from fresh isolated endothelial cells from C57BL/6 mice after LPS or CLP challenge for various days using with RNeasy Mini Kit (Qiagen) followed by DNase treatment. Total RNA $(1 \mu \mathrm{g})$ was reverse-transcribed with Superscript III (Invitrogen) using random primers. Synthesized cDNA samples were amplified in the ABI PRISM 7000 Sequence Detection System (Applied Biosystems) thermocycler using SYBR Green JumpStart Taq ReadyMix (MilliporeSigma). PCR primers are described in Supplemental Table 1. The results of relative expression were normalized to GAPDH mRNA levels in each sample. Results are expressed as mean plus or minus SEM.

Western blotting and immunoprecipitation. Mouse lungs were surgically removed and washed in cold PBS, and homogenized on ice in lysis buffer to prepare tissue lysates. Cell lysates of the freshly isolated endothelial cells were prepared in lysis buffer (50 mM HEPES pH 7.4, $50 \mathrm{mM}$ $\mathrm{NaCl}, 1 \%$ Triton X-100, 5 mM EDTA, 1 mM DTT, 1 mM PMSF, $10 \mu \mathrm{g} /$ $\mathrm{mL}$ aprotinin, $10 \mu \mathrm{g} / \mathrm{mL}$ leupeptin, $10 \mathrm{mM}$ sodium pyrophosphate, 50 $\mathrm{mM}$ sodium fluoride, and $1 \mathrm{mM}$ sodium orthovanadate) for 1 hour. For immunoprecipitation, $500 \mu \mathrm{g}$ total tissue or cell lysates were immunoprecipitated with the primary antibodies and protein A/G PLUS-Agarose beads. Samples were separated by SDS-PAGE, transferred to nitrocellulose membranes, and incubated overnight with the indicated antibodies. After incubation with secondary antibodies, proteins were detected by enhanced chemiluminescence. Quantification of band intensities by densitometry was carried out using ImageJ software.

Liposome-mediated lung endothelial cell-specific gene delivery in vivo. For preparation of liposome $(23,24,52)$, added $315 \mu \mathrm{L}$ DDAB (dimethyl dioctadecyl ammonium bromide), stock solution (400 mg DDAB in $20 \mathrm{~mL}$ chloroform), $200 \mu \mathrm{L}$ cholesterol stock solution (400 mg cholesterol in $20 \mathrm{~mL}$ chloroform), and $9.5 \mathrm{~mL}$ chloroform into the autoclaved round-bottom flask, and swirled everything to mix well. The mixed solution was evaporated using a rotavapor at $37^{\circ} \mathrm{C}$ and $1.85 \mathrm{~g}$ for 30 to 60 minutes. Two milliliters of $5 \%$ glucose was added to the flask to dissolve the lipid. The lipid was sonicated by touching the bottom of the round-bottom flask to the water in the ultrasonic cleaner (2510 Branson) for 30 minutes. The sonicated liposome was transferred from the flask to a polystyrene Eppendorf tube, and sterilized using a $0.45-\mu \mathrm{m}$ filter. Proper amount of plasmid DNA was gently mixed well with the liposome to avoid aggregation (about $50 \mu \mathrm{g}$ of the $2.5-\mathrm{kb}$ $V E$-cadherin promoter-directed CREB expression plasmids and 100 $\mu \mathrm{L}$ liposome) at a total volume of $150 \mu \mathrm{L}$. Liposome-mediated gene delivery was performed by retro-orbital vein injection $(150 \mu \mathrm{L}$ mixture per mice). Seven days later, the expression efficiency and specificity of target genes in tissues and cells were confirmed by Western blot.

Endotoxemia and polymicrobial sepsis models. For LPS-induced endotoxemia sepsis, mice were injected intraperitoneally with a single 
dose of LPS (12 mg/kg) for the indicated time. For the CLP-mediated polymicrobial sepsis, the mouse cecum was ligated below the ileocecal valve with $0.5 \mathrm{~cm}$ ligation length. The cecum was then punctured twice with a 16-gauge needle. Sham control mice were subjected to the same procedure, including opening the peritoneum and exposing the bowel.

sAC activity assay. The activities of SAC (ADCY10) were measured as previously described (19). Briefly, sACs were immunoprecipitated by incubating $20 \mu \mathrm{L}$ polyclonal anti-mouse ADCY10 with $1 \mathrm{~mL}$ total cell lysates (about $1 \mathrm{mg}$ protein) in extraction buffer (50 mM Tris-buffer pH 7.4, $1 \mathrm{mM} \mathrm{EGTA}$, and $1 \mathrm{mM}$ dithiothreitol) with gentle stirring at $4^{\circ} \mathrm{C}$ for 2 hours. Protein $\mathrm{A} / \mathrm{G}$ sepharose beads $(50 \mu \mathrm{L})$ were added and incubated for another 2 hours. The mixture was centrifuged at $425 g$ for 10 minutes, and the pellet was suspended in $3 \mathrm{~mL}$ assay buffer containing 50 mM Tris-HCL buffer pH 7.4, 2 mM DTT, 0.1 mM EGTA, and $1 \mathrm{mM}$ 3-isobutyl-l-xanthine. The reaction was initiated by a fresh addition of $10 \mathrm{mM} \mathrm{MgCl}_{2}$ and $10 \mathrm{mM} \mathrm{ATP}$ at $37^{\circ} \mathrm{C}$ for 20 minutes and stopped by adding $10 \mu \mathrm{L}$ of $0.5 \mathrm{M}$ EDTA to chelate the $\mathrm{Mg}^{2+}$ cofactor. Samples were stored at $-20^{\circ} \mathrm{C}$ for future analysis. Accumulated cAMP was quantified with the cAMP direct immunoassay kit according to the manufacturer's instructions (BioVision). Samples were properly diluted with assay buffer for quantification.

cAMP measurements. Lysates from mouse lung cells were prepared with proper amounts of 0.1 M HCL. Samples were homogenized and centrifuged at top speed for 5 to 10 minutes. Supernatants were then used for cAMP measurement. The levels were determined with a mouse cAMP immunoassay kit (R\&D Systems, KGE012B) according to the manufacturer's instructions. The absorbance at $450 \mathrm{~nm}$ was determined using a 96-well plate reader. Quantification was calculated as $\mathrm{pmol} / \mu \mathrm{g}$ compared with the standard curve.

RNA interference. The mouse CREB (NM_009952.2) and soluble adenylate cyclase 10 (ADCY10, NM_173029.3) SMARTpool siRNA duplexes were synthesized by Dharmacon RNA Technologies. The SMARTpool siRNA is a mixture of 4 different siRNA duplexes against mouse CREB and ADCY1O cDNA sequences. The scrambled siRNA duplex controls were designed and supplied by Dharmacon. Transfection of siRNA duplexes in cells was performed according to the manufacturer's instructions for the Basic Nucleofector Kit for Primary Endothelial Cells (Amaxa Biosystem).

Myeloperoxidase (MPO) assay. MPO activity was performed according to the manufacturer's instructions (Cell Biologics, CB6937). Briefly, lung tissues isolated from mice were homogenized in cold PBS at a ratio of 1:4 (100 $\mathrm{mg}$ tissues to $400 \mathrm{~mL} \mathrm{PBS}$ ) containing proteinase inhibitor (MilliporeSigma cocktail inhibitor 1:100 dilution), and centrifuged at $5000 \mathrm{~g}$ for 5 minutes at $4^{\circ} \mathrm{C}$. Supernatants were removed and the pellets were solved in lysis buffer (50 mM HEPES pH 7.4, 50 $\mathrm{mM} \mathrm{NaCl}, 1 \%$ Triton X-100, 5 mM EDTA, 1 mM DTT, $10 \mathrm{mM}$ sodium pyrophosphate, $50 \mathrm{mM}$ sodium fluoride, and $1 \mathrm{mM}$ sodium orthovanadate) with freshly added proteinase inhibitors, and rotated for 1 hour in a room kept at $4^{\circ} \mathrm{C}$. Samples were centrifuged at $13,000 \mathrm{~g}$ for $10 \mathrm{~min}$ utes at $4^{\circ} \mathrm{C}$ to remove insoluble material. The supernatants (protein samples) were collected for a direct MPO assay. Protein concentration (about 1-2 $\mu \mathrm{g} / \mu \mathrm{L}$ ) was determined by the standard Bio-Rad BCA method. MPO reaction was performed as follows: $50 \mu$ of the protein samples (about 50-100 $\mu \mathrm{g}$ ) were added into $950 \mu \mathrm{L}$ reaction buffer (50 mM phosphate buffer containing $0.167 \mathrm{mg} / \mathrm{mL}$ of o-dianisidine dihydrochloride and $0.0005 \% \mathrm{H}_{2} \mathrm{O}_{2}$ ) for 3-10 minutes. Reaction was stopped by adding $100 \mu \mathrm{L}$ stop buffer $\left(2 \mathrm{M} \mathrm{HCl}\right.$ or $\left.1 \mathrm{M} \mathrm{H}_{2} \mathrm{SO}_{4}\right)$ for 5 minutes. Absorption was measured at $460 \mathrm{~nm}$ to estimate MPO activity. Data were calculated as $\mathrm{A}_{460} / \mathrm{min} / \mathrm{mg}$ protein.

Transwell endothelial permeability measurements. The Transwell permeability assay was done following the kit's instructions (Cell Biologics, CB6929). Briefly, mLMVECs were transfected with CREB expression plasmid using lipofectamine 3000 regents for 2 days. Transfected cells were seeded on the $0.4 \mu \mathrm{m}$ polycarbonate membrane of 6.5-mm Transwell inserts in a 24-well plate, and incubated for 3 days until full confluent monolayers were formed. IL-1 $\beta(2 \mathrm{ng} / \mathrm{mL})$ and HRP $(100 \mu \mathrm{g} / \mathrm{mL})$ were added into starved cells in Transwell inserts filled with serum-free medium. Cells were incubated for the indicated time for permeability analysis. Media $(20 \mu \mathrm{L})$ from the lower chamber was transferred to a 96-well ELISA plate. TMB substrate $(50 \mu \mathrm{L})$ was added into the wells, and left for 5 minutes until the solution turned blue. Spectrophotometric absorbance analysis at $450 \mathrm{~nm}$ provided a quantitative evaluation of the amount of HRP that was leaked from the disrupted barrier of endothelial cells. Data are presented as mean \pm SEM of at least 3 independent experiments performed in triplicate.

Intravital lung imaging of polymorphonuclear leukocytes (PMNs) in lung microvessels. Surgical methods for gaining access to lungs were previously described (22). We injected Brilliant Violet 421-labeled LY6G antibody (10 $\mu \mathrm{g} / \mathrm{mice})$ (clone $1 \mathrm{~A} 8$, BioLegend) via jugular vein as well as SeTau647-labeled (SETA BioMedicals) CD31 antibody (20 $\mu \mathrm{g} / \mathrm{mice})$ (clone 390, BioLegend) to stain PMNs and lung vessel endothelium, respectively, before the surgery. A resonant-scanning 2-photon Ultima Multiphoton Microscope (Bruker) with an Olympus XLUMPlanFL N $\times 20$ (NA 1.00) was used to collect dual-color images (emission filter; 460/50 nm for Brilliant Violet 421, 708/75 nm for SeTau647) with $820 \mathrm{~nm}$ excitation. Images were processed and analyzed by Image J and customized LabVIEW programs.

Lung vascular permeability measurements. Evans blue-albumin pulmonary transvascular flux measurements were performed to measure vessel endothelial permeability $(8,53)$. Briefly, $150 \mu \mathrm{L}$ Evans blue-albumin (1\% Evans blue dye [EBD] and $4 \%$ albumin in PBS) was injected into anesthetized mice and allowed to circulate in the blood vessels for 30 minutes. Mice were scarified and lungs were perfused by PBS for 2 minutes. Lung tissues were then excised, weighed, homogenized in $1 \mathrm{~mL} \mathrm{PBS}$ and extracted overnight in $2 \mathrm{~mL}$ formamide at $60^{\circ} \mathrm{C}$. Evans blue concentration in lung homogenate supernatants was quantified by the spectrophotometric method at absorbance of $620 \mathrm{~nm}$ and $740 \mathrm{~nm}$. Tissue EBD content ( $\mu \mathrm{g} \mathrm{EBD/g}$ fresh lung tissue) was calculated by comparing tissue supernatant $\mathrm{A}_{620}$ readings with an EBD standard curve. Concentration of Evans blue dye was determined in micrograms per gram of lung tissue. The ratio of wet lung to dry lung weight was calculated.

ELISA. The levels of IL-1 $\beta$, IL-18, and TNF- $\alpha$ in mouse serum were determined by Quantikine sandwich ELISA kits (R\&D Systems). Mouse IL-18 levels were measured with the MBL ELISA kit. The cytokine concentration was measured at $450 \mathrm{~nm}$ wavelength of absorbance, and calculated by GraphPad Prism linear regression analysis.

Patient samples. Seven ARD patients (4 males, 3 females) and 7 cardiogenic pulmonary edema (CPE) patients (5 males, 2 females) from the intensive care unit of the Guangdong General Hospital who received mechanical ventilation for ARD and CPE were enrolled. Ten healthy volunteers were included as controls. The levels of cytokine IL-1 1 , IL-1RA, and cAMP levels in plasma were measured by ELISA. Data on demographic characteristics and medical history were previ- 
ously described (8). A total of 10 stable outpatients ( 7 males, 3 females) were also enrolled as a control group.

Statistics. Data were analyzed by 2-tailed unpaired Student's $t$ test for comparisons of 2 groups or 1-way ANOVA of the repeated experiments, followed by the Tukey's post hoc pairwise multiple comparisons when appropriate with Prism 7 (GraphPad Software Inc.). A $P$ value of less than 0.05 was considered significant. For all bar graphs, the mean \pm SEM was plotted unless otherwise indicated. All in vitro experiments were repeated at least 3 times unless otherwise indicated.

Study approval. Animal experiments and euthanasia protocols were performed in strict compliance with the NIH guidelines for humane treatment and approved by the University of Illinois (Chicago, Illinois, USA) Animal Care and Use Committee. The human study was approved by the Medical Ethics Committee of the Guangdong Provincial People's Hospital (Guangzhou, China). Written informed consent was required from participants or their surrogates.

\section{Author contributions}

ABM conceived the study. SX designed the research, drafted the manuscript, and performed the experiments and analyzed data with help from and discussions with JR, ZH, LSH, YT, SN, AD, MZ, WW, ZY, XG, and DM. GNR provided CREB floxed mice. ABM and JR directed the study and critically reviewed and revised the manuscript.

\section{Acknowledgments}

This work was supported by NIH grants P01-HL60678, P01HL077806, T32-HL007829, R01-HL118068, and R01-HL90152.

Address correspondence to: Asrar B. Malik, Department of Pharmacology, University of Illinois College of Medicine, 835 South Wolcott Avenue, Chicago, Illinois 60612, USA. Phone: 312.996.7635; Email: abmalik@uic.edu.
1. Matthay MA, et al. Acute respiratory distress syndrome. Nat Rev Dis Primers. 2019;5(1):18.

2. Polat G, Ugan RA, Cadirci E, Halici Z. Sepsis and septic shock: current treatment strategies and new approaches. Eurasian JMed. 2017;49(1):53-58.

3. Lee WL, Slutsky AS. Sepsis and endothelial permeability. N Engl J Med.2010;363(7):689-691.

4. Matthay MA, Ware LB, Zimmerman GA. The acute respiratory distress syndrome. J Clin Invest. 2012;122(8):2731-2740.

5. Hoyer FF, et al. Tissue-specific macrophage responses to remote injury impact the outcome of subsequent local immune challenge. Immunity. 2019;51(5):899-914.e7.

6. Di A, et al. The TWIK2 potassium efflux channel in macrophages mediates NLRP3 inflammasome-induced inflammation. Immunity. 2018;49(1):56-65.e4.

7. Erdei J, et al. Induction of NLRP3 inflammasome activation by heme in human endothelial cells. Oxid Med Cell Longev. 2018;2018:4310816.

8. Cheng KT, et al. Caspase-11-mediated endothelial pyroptosis underlies endotoxemia-induced lung injury. J Clin Invest. 2017;127(11):4124-4135.

9. Evavold CL, Kagan JC. Inflammasomes: threat-assessment organelles of the innate immune system. Immunity. 2019;51(4):609-624.

10. Liu X, et al. Cell-type-specific interleukin 1 receptor 1 signaling in the brain regulates distinct neuroimmune activities. Immunity. 2019;50(2):317-333.e6.

11. Zhu W, et al. Interleukin receptor activates a MYD88-ARNO-ARF6 cascade to disrupt vascular stability. Nature. 2012;492(7428):252-255.

12. Komarova YA, Kruse K, Mehta D, Malik AB. Protein interactions at endothelial junctions and signaling mechanisms regulating endothelial permeability. Circ Res. 2017;120(1):179-206.

13. Wettschureck N, Strilic B, Offermanns S. Passing the vascular barrier: endothelial signaling processes controlling extravasation. Physiol Rev. 2019;99(3):1467-1525.

14. Orsenigo F, et al. Phosphorylation of VE-cadherin is modulated by haemodynamic forces and contributes to the regulation of vascular permeability in vivo. Nat Commun. 2012;3:1208.

15. Nanes BA, et al. p120-catenin regulates VE-cadherin endocytosis and degradation induced by the Kaposi sarcoma-associated ubiquitin ligase K5. Mol Biol Cell. 2017;28(1):30-40.

16. Friedrich EE, et al. Endothelial cell Piezo1 mediates pressure-induced lung vascular hyperpermeability via disruption of adherens junctions. Proc Natl Acad Sci USA. 2019;116(26):12980-12985.

17. Gong $\mathrm{H}$, et al. HIF2 $\alpha$ signaling inhibits adherens junctional disruption in acute lung injury. J Clin Invest. 2015;125(2):652-664.

18. Kim SH, et al. Tunable regulation of CREB DNA binding activity couples genotoxic stress response and metabolism. Nucleic Acids Res. 2016;44(20):9667-9680.

19. Chen Y, et al. Soluble adenylyl cyclase as an evolutionarily conserved bicarbonate sensor. Science. 2000;289(5479):625-628.

20. Park SJ, et al. Resveratrol ameliorates agingrelated metabolic phenotypes by inhibiting cAMP phosphodiesterases. Cell. 2012;148(3):421-433.

21. Conti M, Beavo J. Biochemistry and physiology of cyclic nucleotide phosphodiesterases: essential components in cyclic nucleotide signaling. Annu Rev Biochem. 2007;76:481-511.

22. Looney MR, Thornton EE, Sen D, Lamm WJ, Glenny RW, Krummel MF. Stabilized imaging of immune surveillance in the mouse lung. Nat Methods. 2011;8(1):91-96.

23. Zhou MY, et al. In vivo expression of neutrophil inhibitory factor via gene transfer prevents lipopolysaccharide-induced lung neutrophil infiltration and injury by a beta 2 integrin-dependent mechanism. JClin Invest. 1998;101(11):2427-2437.

24. Liu M, et al. Sox17 is required for endothelial regeneration following inflammation-induced vascular injury. Nat Commun. 2019;10(1):2126.

25. Liu YC, Yu MM, Shou ST, Chai YF. Sepsisinduced cardiomyopathy: mechanisms and treatments. Front Immunol. 2017;8:1021.

26. London NR, et al. Targeting Robo4-dependent Slit signaling to survive the cytokine storm in sepsis and influenza. Sci Transl Med. 2010;2(23):23ra19.

27. Lampugnani MG, Dejana E, Giampietro C. Vascular endothelial (VE)-cadherin, endothelial adherens junctions, and vascular disease. Cold Spring Harb Perspect Biol. 2018;10(10):a029322.

28. Ohmura $\mathrm{T}$, et al. Regulation of lung endothelial permeability and inflammatory responses by prostaglandin A2: role of EP4 receptor. Mol Biol Cell. 2017;28(12):1622-1635.

29. Noval Rivas M, et al. Intestinal permeability and IgA provoke immune vasculitis linked to cardiovascular inflammation. Immunity. 2019;51(3):508-521.e6.

30. Ridker PM, et al. Antiinflammatory therapy with canakinumab for atherosclerotic disease. $N$ EnglJ Med. 2017;377(12):1119-1131.

31. Verma S, Leiter LA, Bhatt DL. CANTOS ushers in a new calculus of inflammasome targeting for vascular protection-and maybe more. Cell Metab. 2017;26(5):703-705.

32. Chaudhry H, et al. Role of cytokines as a double-edged sword in sepsis. In Vivo. 2013;27(6):669-684.

33. Fisher CJ, et al. Recombinant human interleukin 1 receptor antagonist in the treatment of patients with sepsis syndrome. Results from a randomized, double-blind, placebo-controlled trial. Phase III rhIL-1ra Sepsis Syndrome Study Group. JAMA. 1994;271(23):1836-1843.

34. Meyer NJ, et al. IL1RN coding variant is associated with lower risk of acute respiratory distress syndrome and increased plasma IL-1 receptor antagonist. Am J Respir Crit Care Med. 2013;187(9):950-959.

35. Meyer NJ, et al. A functional synonymous coding variant in the IL1RN gene is associated with survival in septic shock. Am J Respir Crit Care Med. 2014;190(6):656-664.

36. Park WY, et al. Cytokine balance in the lungs of patients with acute respiratory distress syndrome. Am J Respir Crit Care Med. 2001;164(10 Pt 1):1896-1903.

37. Meyer NJ, et al. Mortality benefit of recombinant human interleukin-1 receptor antagonist for sepsis varies by initial interleukin-1 receptor antagonist plasma concentration. Crit Care Med. 2018;46(1):21-28.

38. Evavold CL, Ruan J, Tan Y, Xia S, Wu H, Kagan JC. The pore-forming protein gasdermin $\mathrm{D}$ regulates interleukin-1 secretion from living macrophages. Immunity. 2018;48(1):35-44.e6.

39. Rühl S, Shkarina K, Demarco B, Heilig R, Santos JC, Broz P. ESCRT-dependent mem- 


\section{RESEARCH ARTICLE}

brane repair negatively regulates pyroptosis downstream of GSDMD activation. Science. 2018;362(6417):956-960.

40. Chava KR, Tauseef M, Sharma T, Mehta D. Cyclic AMP response element-binding protein prevents endothelial permeability increase through transcriptional controlling p190RhoGAP expression. Blood. 2012;119(1):308-319.

41. Korayem AH, et al. Endothelial cAMP deactivates ischemia-reperfusion-induced microvascular hyperpermeability via Rap1-mediated mechanisms. Am J Physiol Heart Circ Physiol. 2017;313(1):H179-H189.

42. Ramos CJ, Lin C, Liu X, Antonetti DA. The EPACRap1 pathway prevents and reverses cytokineinduced retinal vascular permeability. J Biol Chem. 2018;293(2):717-730.

43. Lefkimmiatis K, Zaccolo M. cAMP signaling in subcellular compartments. Pharmacol Ther.
2014;143(3):295-304.

44. Waschke J, Drenckhahn D, Adamson RH, Barth H, Curry FE. cAMP protects endothelial barrier functions by preventing Rac-1 inhibition. Am JPhysiol Heart Circ Physiol. 2004;287(6):H2427-H2433.

45. Sayner SL, Alexeyev M, Dessauer CW, Stevens T. Soluble adenylyl cyclase reveals the significance of cAMP compartmentation on pulmonary microvascular endothelial cell barrier. Circ Res. 2006;98(5):675-681.

46. Fischmeister R. Is cAMP good or bad? Depends on where it's made. Circ Res. 2006;98(5):582-584.

47. Schlegel N, Waschke J. Impaired cAMP and Rac 1 signaling contribute to TNF-alpha-induced endothelial barrier breakdown in microvascular endothelium. Microcirculation. 2009;16(6):521-533.

48. Johnstone TB, Agarwal SR, Harvey RD, Ostrom RS. cAMP signaling compartmentation: adenylyl cyclases as anchors of dynamic signaling com- plexes. Mol Pharmacol. 2018;93(4):270-276.

49. Kimmoun A, Novy E, Auchet T, Ducrocq N, Levy B. Hemodynamic consequences of severe lactic acidosis in shock states: from bench to bedside. Crit Care. 2015;19:175.

50. Kleinboelting S, et al. Crystal structures of human soluble adenylyl cyclase reveal mechanisms of catalysis and of its activation through bicarbonate. Proc Natl Acad Sci USA. 2014;111(10):3727-3732.

51. Lakhina V, et al. Genome-wide functional analysis of CREB/long-term memory-dependent transcription reveals distinct basal and memory gene expression programs. Neuron. 2015;85(2):330-345.

52. Fisher RK, et al. Improving the efficacy of liposomemediated vascular gene therapy via lipid surface modifications. J Surg Res. 2017;219:136-144.

53. Radu M, Chernoff J. An in vivo assay to test blood vessel permeability. JVis Exp. 2013;(73):e50062. 